\title{
Analysis of BSRT profiles in the LHC at injection*
}

\author{
M. Fitterer ${ }^{\dagger}$ and G. Stancari \\ Fermi National Accelerator Laboratory, PO Box 500, Batavia, Illinois 60510, USA
}

S. Papadopoulou, Y. Papaphilippou, S. Redaelli

CERN, CH-1211 Geneva 23, Switzerland

(Dated: December 20, 2017)

\begin{abstract}
The beam synchrotron radiation telescope (BSRT) at the LHC allows to take profiles of the transverse beam distribution, which can provide useful additional insight in the evolution of the transverse beam distribution. A python class has been developed [1], which allows to read in the BSRT profiles, usually stored in binary format, run different analysis tools and generate plots of the statistical parameters and profiles as well as videos of the the profiles. The detailed analysis will be described in this note. The analysis is based on the data obtained at injection energy (450 GeV) during MD1217 [2] and MD1415 [3] which will be also used as illustrative example. A similar approach is also taken with a MATLAB based analysis described in [4].
\end{abstract}

\footnotetext{
* Fermilab is operated by Fermi Research Alliance, LLC under Contract No. DE-AC02-07CH11359 with the United States Department of Energy. This work was partially supported by the US DOE LHC Accelerator Research Program (LARP) and by the European FP7 HiLumi LHC Design Study, Grant Agreement 284404.

$\dagger$ Email:〈mfittere@fnal.gov $\rangle$.
} 


\section{CONTENTS}

$\begin{array}{ll}\text { I. Introduction } & 3\end{array}$

II. Pre-processing of BSRT data 5

A. Discarding profiles without data $\quad 5$

B. Normalizing and averaging of profiles $\quad 6$

$\begin{array}{ll}\text { C. Background subtraction } & 7\end{array}$

1. Estimate and removal of background 8

2. Comparison with background estimate from Gaussian and q-Gaussian fit 9

3. Influence of background subtraction on statistical parameters 10

III. Calculation of statistical parameters 11

A. Asymmetry of BSRT profiles 11

B. Gaussian and q-Gaussian fit $\quad 12$

C. Statistical estimate of beam centroid, beam sigma and beam halo 17

1. Beam centroid and beam sigma 17

2. Beam halo 21

3. Conversion to beam sigma and emittance 23

D. Residual and ratio of profiles 23

$\begin{array}{ll}\text { IV. Summary } & 25\end{array}$

$\begin{array}{ll}\text { Acknowledgments } & 25\end{array}$

$\begin{array}{ll}\text { References } & 26\end{array}$ 


\section{INTRODUCTION}

The transverse beam distribution in the LHC is in general non-Gaussian and for many different studies a knowledge of the exact beam distribution and in particular also the population of the tails is needed. The beam synchrotron radiation telescope (BSRT), with its ability to also take beam profiles, is currently the best available tool for this purpose. In the future, the installation of several other beam instrumentation devices for in particular halo diagnostics is foreseen or new device are currently being developed [5].

This note describes the analysis of the BSRT profiles as implemented in [1]. The software is open source and can be downloaded from the github repository:

https://github.com/mfittere/pytimbertools

Documentation is done in this note and in the docstrings. The analysis was developed based on the profiles taken at injection with $E_{\text {beam }}=450 \mathrm{GeV}$ in MD1217 [2] and MD1415 [3]. For simplicity but without loss of generality, we will only show plots for the reference bunches during these two MDs, meaning bunches without excitation. The beam parameters for these bunches are listed in Table I.

Table I. Beam parameters for references bunches during MD1217 [2] and MD1415 [3].

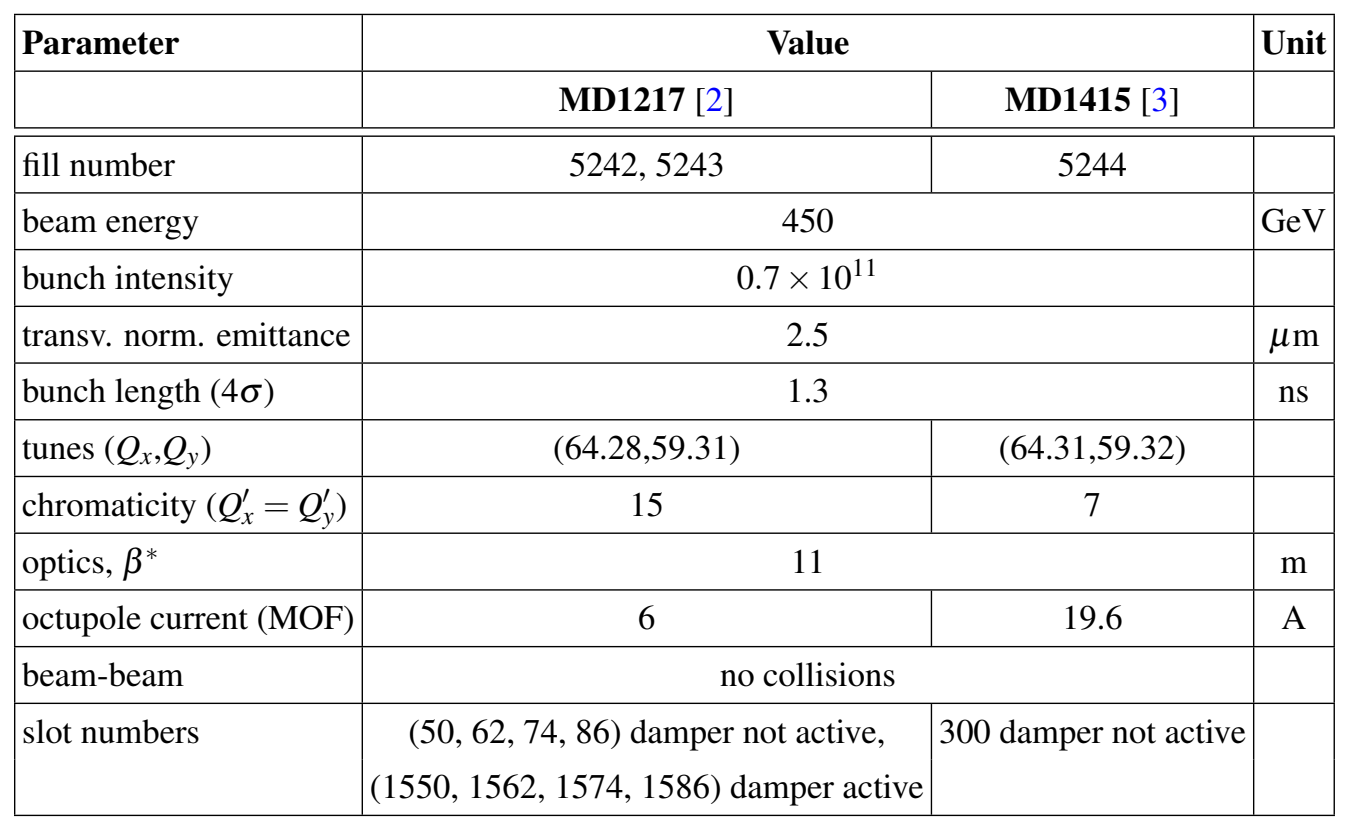

A different analysis approach might have to be taken at top energy with $E_{\text {beam }}=6.5 \mathrm{TeV}$ or $7.0 \mathrm{TeV}$ as the profiles then are limited mainly by diffraction.

A typical analysis of the profiles follows the following steps:

1. Read in profiles: Profiles are saved in binary format and are read in and stored in a dictionary:

myprofiles $=$ BSRTprofiles.load_files(files='LHC.BSRTS.5R4.B1_profiles.bindata') 
2. Discard profiles containing only noise: All profiles containing only noise are deleted from the dictionary of the BSRT class containing the raw profiles:

myprofiles.clean_data()

3. Normalize and average profiles: All profiles are converted to a probability distribution with integral of 1 over the distribution:

myprofiles.norm(nmvavg=10)

This profiles will be referred to in this note as "normalized profiles". In the profiles analyzed in this note, three profiles are taken for each time stamp. After normalization the average over the profiles with the same time stamp (here 3 profiles) is taken and stored in self .profiles_norm_avg and the moving average over all profiles with the nmvavg previous/following time stamps (here in total $(10+1) \cdot 3=33$ profiles) is taken and stored in self .profiles_norm_mv_avg.

4. Remove background: Optionally the background of the normalized profiles can be removed or respectively reduced by one order of magnitude:

myprofiles.remove_background()

Usually this step did not prove to be necessary or too useful.

5. Calculation of statistical parameters: Different statistical parameters are calculated and the distribution is fitted with a Gaussian and q-Gaussian distribution (see Sec. III) for the averaged profiles (self.profiles_norm_avg) and moving average profiles (self.profiles_norm_mv_avg):

myprofiles.get_stats (beam='b1', db=db)

The data is then stored in self .profiles_norm_avg_stat and self .profiles_norm_mvavg_stat. To obtain the emittances the standard deviation of the profiles has to be converted to the beam emittance. By providing a pytimber database with the beam energy (LHC.STATS:ENERGY), the BSRT LSF-factor (LHC.BSRT.5[LR]4.B[12]:LSF $\backslash_{-}[\mathrm{HV}]$ ) and the $\beta$-function at the BSRT (LHC.BSRT . 5 [LR] 4.B [12] :BETA \_ [HV]), the conversion is done automatically.

6. Plot profiles and statistical parameters: A typical plot of the BSRT profiles as obtained with myprofiles.plot_all (mvavg=True, ... )

is shown in Fig. 1. It includes the plot of the normalized profiles, the residual and ratio and the cumulative sum (see Sec. III). The same plots can also be arranged as a video with the command: 

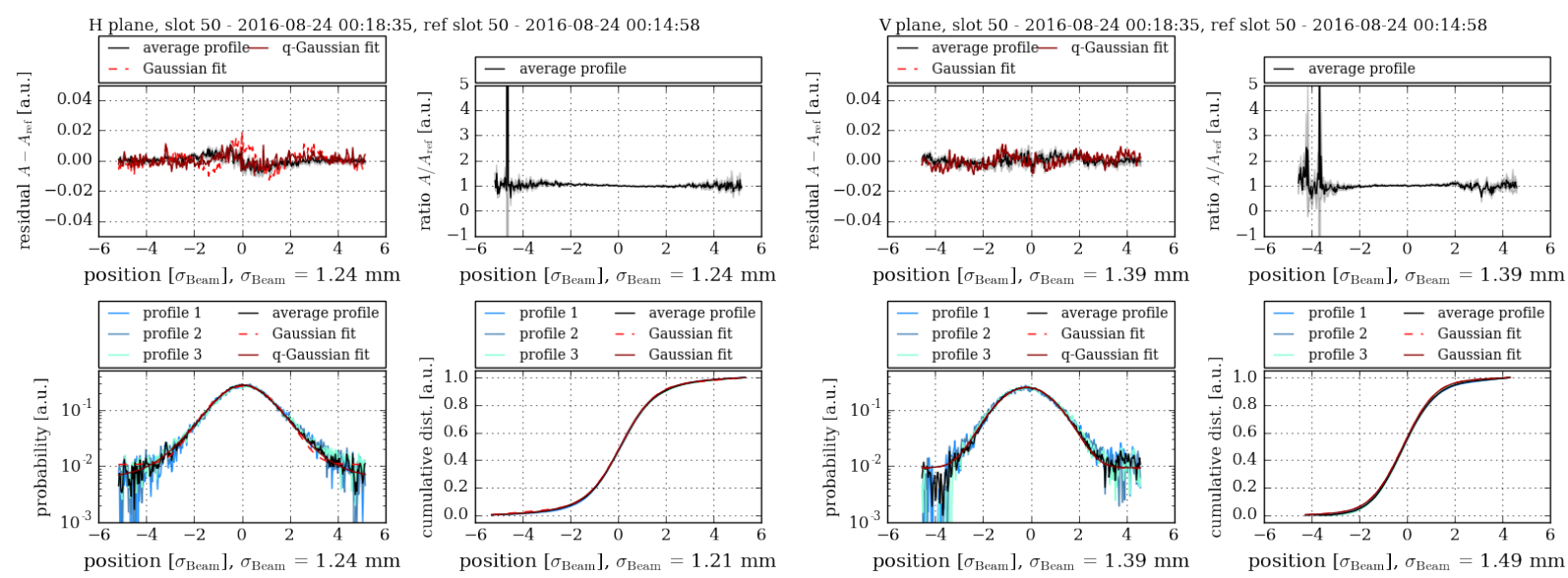

Figure 1. Typical BSRT profile plot at injection $(450 \mathrm{GeV})$ during fill 5242 and slot 50 in the horizontal (left) and vertical (right) plane. The moving averaged profile over $3 \cdot 11$ profiles is shown in black and the residual and ratio as well as the fits are obtained using the moving averaged profile.

myprofiles.mk_profile_video(mvavg=True...)

In addition, also the evolution of the statistical parameters during the fill can be plotted with:

myprofiles.plot_stats (...)

\section{PRE-PROCESSING OF BSRT DATA}

Here we describe which profiles are discarded (self.clean_data(), Sec. II A), the normalization of the profiles (self.norm(), Sec. II B), and the removal of the background (self.remove_background(), Sec. II C).

\section{A. Discarding profiles without data}

Some profiles just consist of noise. For illustration Fig. 2 shows a normal profile and a discarded profile. These profiles can be removed from the data with the command:

self. clean_data (stdamp=3000)

The criterion is that the standard deviation of the profile amplitude (stdamp) is smaller than a user given value, here usually 3000. Alternatively one could have also set a limit on the peak amplitude. However, we considered the standard variation more robust as the peak of the profile could vary considerably depending on the bunch intensity while the standard deviation in case of just noise stayed rather constant also for different bunch intensities. 

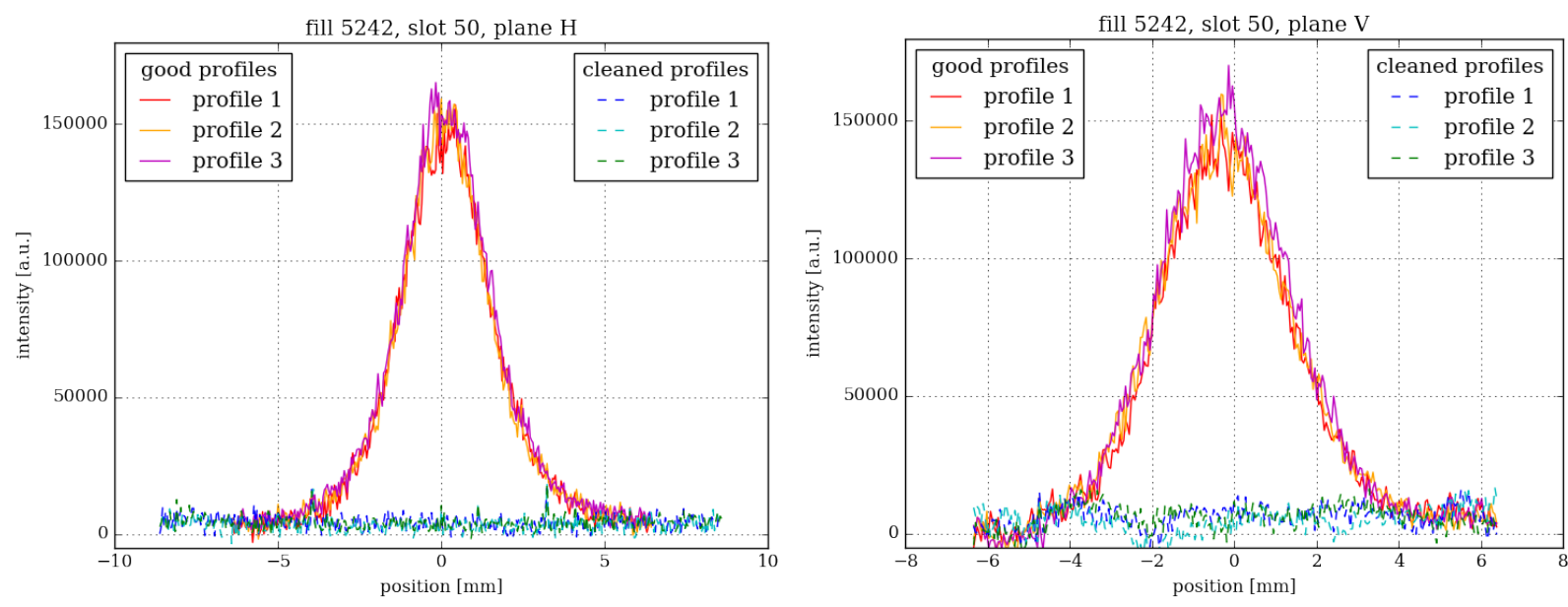

Figure 2. Example of BSRT profiles at injection $(450 \mathrm{GeV})$ during fill 5242 and slot 50 in the horizontal (left) and vertical (right) plane. The good profiles taken at 2016-08-24 00:18:35 are shown in red colors and the profile which are just noise taken at 2016-08-24 03:04:37 are shown in blue colors.

\section{B. Normalizing and averaging of profiles}

As the profile amplitude can vary considerably between the different profiles, it is useful to normalize the profiles in order to be able to later average over several profiles. Both steps, normalization and averaging, are performed by calling:

self. norm (nmvavg=10)

where nmvavg is the number of profiles used for the moving average (see later in this section). In this analysis, we chose to normalize the distribution with the integral over the distribution:

$$
A_{i, \text { normalized }}=\frac{A_{i}}{\sum_{i=\text { bins }} A_{i} \cdot \Delta z_{i}}, \quad z=x, y
$$

where $A_{i}$ is the amplitude of bin $i$ and $\Delta z_{i}$ the bin width. For the analyzed profiles the bin width is constant and the same in both planes with $\Delta z_{i}=0.0431 \mathrm{~mm}$. With this definition the integral over the normalized profile distributions $\rho(z)$ :

$$
\int_{-\infty}^{+\infty} \rho(z) d z \approx \sum_{i=\text { bins }} A_{i, \text { normalized }} \cdot \Delta z_{i}=1, \quad z=x, y
$$

is 1 and the distribution can then be also seen as a probability distribution with the correct normalization. For illustration the profiles before and after normalization are shown in Fig. 3.

After normalization, the average over all profiles with the same time stamp (usually 3 profiles) is taken and stored in self.profiles_norm_avg. In addition, the moving average over nmvavg+1 timestamps (here $(10+1) \cdot 3$ profiles) is taken and stored in self .profiles_norm_mvavg (black line in Fig. 3). Explicitly, the average profile is taken by calculating the mean value of the amplitude $A_{i}$ for each bin over all 

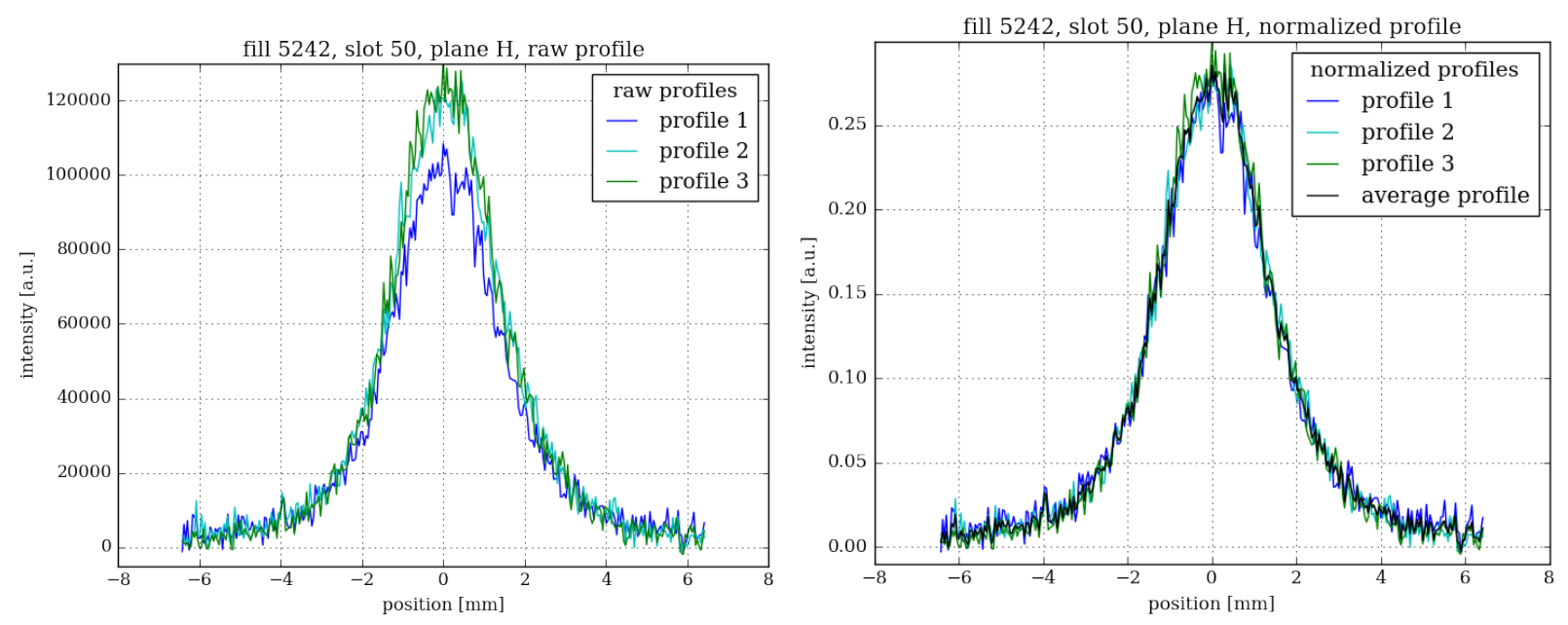

Figure 3. BSRT profiles at injection (450 GeV) during fill 5242 at 2016-08-24 00:21:20 for slot 50 in the horizontal plane before (left) and after (right) normalization.

$n_{\text {prof profiles }}$

$$
\mu_{A_{i}}=\frac{\sum_{j=1}^{n_{\text {prof }}} A_{i, j}}{n_{\text {prof }}}, \quad i=1, \ldots, n_{\mathrm{bins}},
$$

which defines the amplitude of the average/moving average profile for each bin. The noise on each bin can then be estimated by the standard deviation (e.g. self.profiles_norm_avg['ampstd'])

$$
\sigma_{A_{i}}=\sqrt{\frac{1}{n_{\mathrm{prof}}} \sum_{j=1}^{n_{\mathrm{prof}}}\left(A_{i, j}-\mu_{A_{i}}\right)^{2}}, \quad i=1, \ldots, n_{\mathrm{bins}}
$$

yielding also the error on the mean value $\mu_{A_{i}}$ (e.g. self.profiles_norm_avg ['amperr'])

$$
\sigma_{\mu_{A_{i}}}=\frac{\sigma_{A_{i}}}{n_{\mathrm{prof}}}
$$

The standard deviation $\sigma_{A_{i}}$ is later used to define the uncertainties for the Gaussian and q-Gaussian fit (see Sec. III B). The error on the mean value $\sigma_{\mu_{A_{i}}}$ is needed for the calculation of the error of the residual and ratio (see Sec. III D).

\section{Background subtraction}

To properly remove the background several profiles without beam would ideally have to be taken before the actual measurements with beam. From the profiles without beam a proper model of the background could then be extracted, but unfortunately no profiles without beam were taken. 


\section{Estimate and removal of background}

As the profiles without beam are not available, the background is instead estimated by taking the average over the bgnavg (default 10) outermost bins for each slot and timestamp:

self.remove_background (bgnavg $=10)$

This average will be abbreviated with $B_{\text {left,slot }}(t), B_{\text {right,slot }}(t)$ and $B_{\text {slot }}(t)=\operatorname{mean}\left(B_{\text {left,slot }}(t), B_{\text {right,slot }}(t)\right)$. This estimate of the background fluctuates in general around some average value (see Fig. 4).
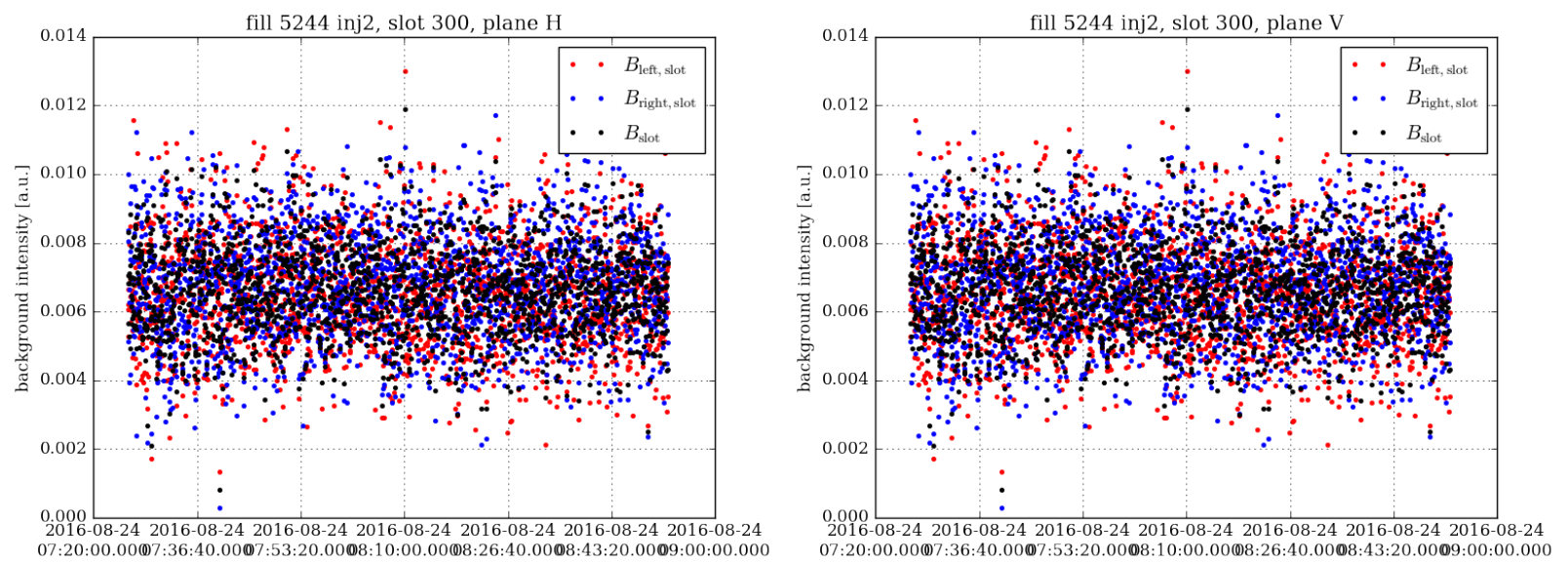

Figure 4. Estimate of background of BSRT profiles at injection $(450 \mathrm{GeV})$ during fill 5244 for slot 300 in the horizontal (left) and vertical (right) plane (average profiles). The background fluctuates around an average value and there are no considerable differences between averaging over the left $\left(B_{\text {left,slot }}(t)\right)$ or right $\left(B_{\text {right,slot }}(t)\right)$ outermost bins.

A robust and global estimate for the background is therefore the average value of $B_{\text {left,slot }}(t), B_{\text {right,slot }}(t)$ over all time stamps for each slot:

$$
B_{\text {global }, \text { slot }}=\sum_{\mathrm{t}_{\mathrm{i}}=\text { all time stamps }} \frac{B_{\text {left,slot }}\left(t_{i}\right)+B_{\text {right,slot }}\left(t_{i}\right)}{2}
$$

Explicitly, this returns one constant value for each slot. This constant value $B_{\text {global,slot }}$ for each slot is then subtracted from all profiles and the profiles are afterwards renormalized. This definition particularly avoids that the statistical parameters like e.g. the beam sigma are dominated by the fluctuations of the background itself. An example of the profiles before and after renormalization is shown in Fig. 5, which shows a reduction of the background level by approximately one order of magnitude. If absolute values of the beam sigma, centroid etc. and also the Gaussian and q-Gaussian fit are required, it is better to subtract the background as it considerably contributes to the magnitude of the values. If only relative changes with respect to e.g. the initial values are needed, it is better to not subtract the background. In both cases it is always useful to also look at the evolution of the background bg_avg_left, bg_avg_right and bg_avg as sometimes jumps occur which can then in turn cause jumps in other statistical parameters like the beam sigma. 

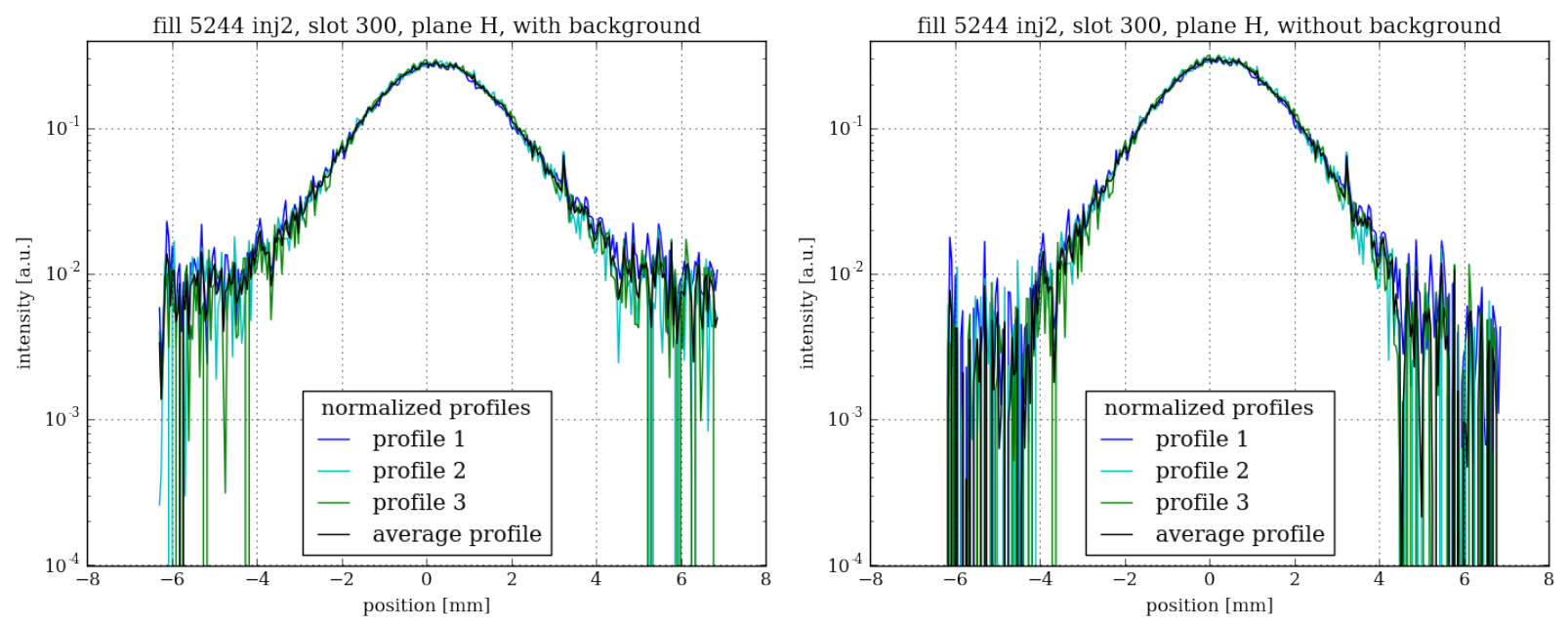

Figure 5. BSRT profiles at injection (450 GeV) during fill 5242 at 2016-08-24 07:27:05 for slot 300 in the horizontal plane before (left) and after (right) background subtraction. The plot shows the normalized profile in green/blue and the average profile in black.

\section{Comparison with background estimate from Gaussian and q-Gaussian fit}

Alternatively the background can also be estimated by the parameter $c$ introduced in the Gaussian

$$
f_{\text {fit }}(z):=c_{\text {Gauss }}+a_{\text {Gauss }} \cdot f_{\text {Gauss }}(z, \mu, \sigma), z=x, y,
$$

and equivalent in the q-Gaussian fit

$$
f_{\text {fit }}(z):=c_{\mathrm{q}-\text { Gauss }}+a_{\mathrm{q}-\text { Gauss }} \cdot f_{\mathrm{q}-\text { Gauss }}(z, q, \mu, \beta), z=x, y .
$$
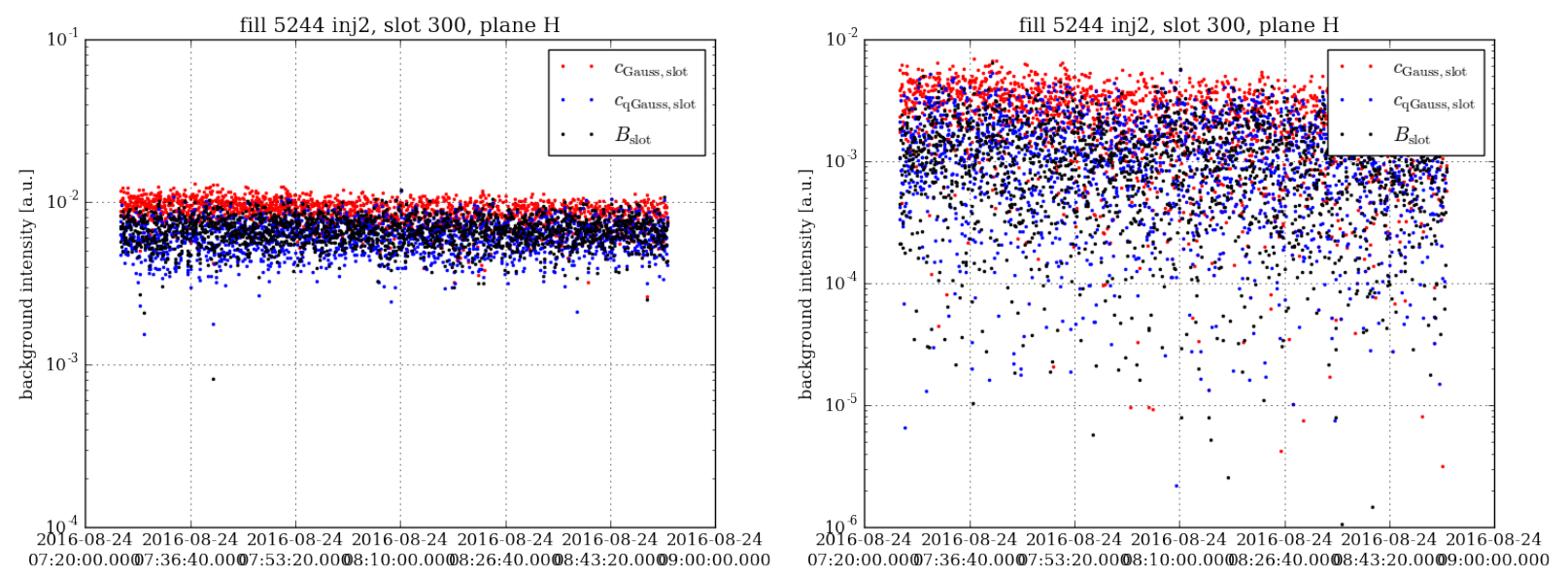

Figure 6. Comparison of the different background estimates of the BSRT profiles at injection $(450 \mathrm{GeV})$ during fill 5244 for slot 300 in the horizontal plane with background (left) and with the background subtracted (right) as described in Sec. IIC 1. $B_{\text {slot }}$ denotes the background estimate by averaging the left and right outermost bins (see Sec. II C 1) and $c_{\text {Gauss,slot }}$ and $c_{\mathrm{qGauss}, \text { slot }}$ are the fit parameters from the Gaussian and q-Gaussian fit respectively (see Sec. III B). Here the values for the average profiles are shown. 
to account for the background in the profiles (see Sec. III B). Here with $\mu$ and $\sigma$ are the mean and standard variation of the distribution and $\beta$ and $q$ the additional fit parameters of the q-Gaussian distribution related to the standard variation. Fig. 6 shows a comparison of the three estimates with and without background reduced. All values lie around approximately the same average value confirming that the estimate by taking the average over the left and right outermost bins is a good and robust estimate. All three estimates for the background could in general be used. We decided to use the averaging over the left/right outermost bins as it presents a model independent estimate in contrast to the Gaussian and q-Gaussian fit which both assume a certain beam distribution.

\section{Influence of background subtraction on statistical parameters}

The background can contribute considerably to the calculation of the statistical parameters. As example, the standard deviation $\sigma$ of the profiles obtained with three different methods (see Sec. III) - statistical rms $\operatorname{sum}\left(\sigma_{\text {stat }}\right)$, Gaussian fit ( $\left.\sigma_{\text {Gauss }}\right)$ and q-Gaussian fit $\left(\sigma_{\mathrm{qGauss}}\right)-$ is shown in Fig. 7, where the statistical rms sum $\sigma_{\text {stat }}$ is defined by (Eqn. 25):

$$
\begin{aligned}
& \mu_{\text {stat }}=\frac{\sum_{i=\text { bins }} z_{i} \cdot A_{i}}{\sum_{i=\mathrm{bins}} \cdot A_{i}}, z=x, y, \\
& \sigma_{\text {stat }}=\sqrt{\frac{\sum_{i=\text { bins }} A_{i} \cdot\left(z_{i}-\mu_{\text {stat }}\right)^{2}}{\sum_{i=\text { bins }} \cdot A_{i}}}
\end{aligned}
$$

The absolute value of the statistical rms sum $\sigma_{\text {stat }}$ is heavily influenced by the background of the profiles as it does not include any parameter to account for the background. This behavior is typical for any statistical
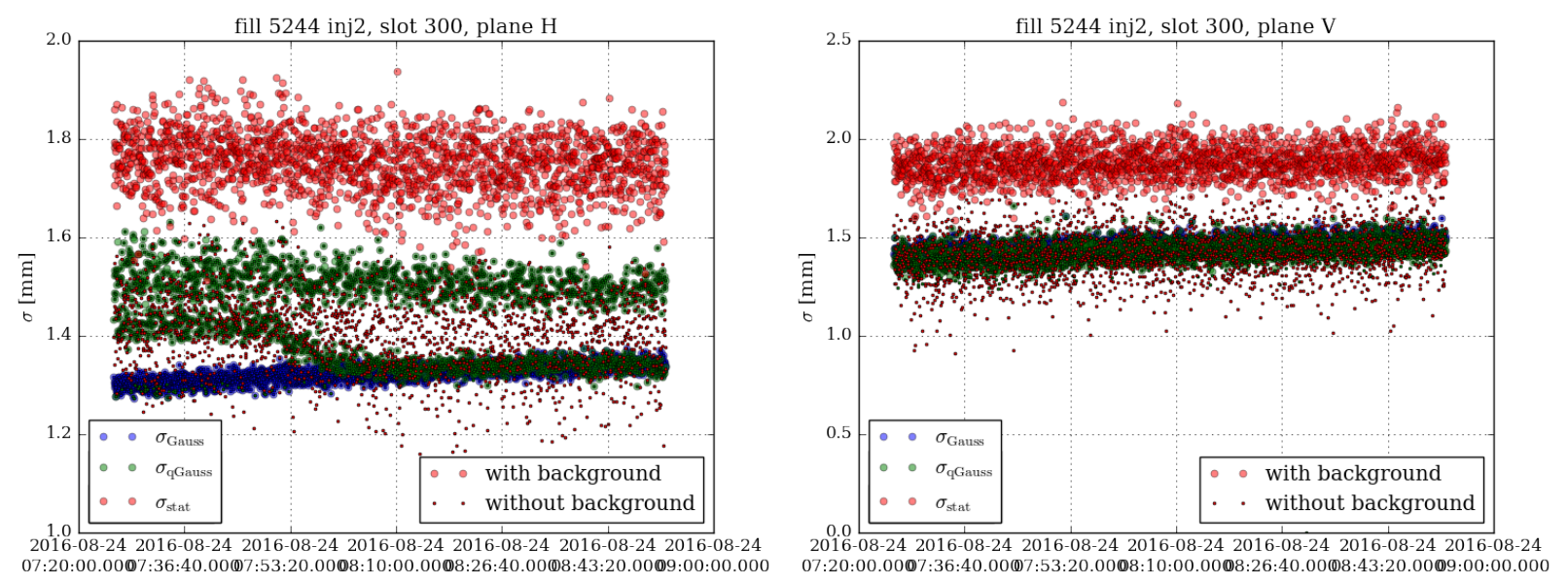

Figure 7. Standard deviation $\sigma$ of BSRT profiles at injection $(450 \mathrm{GeV})$ during fill 5244 for slot 300 in the horizontal (left) and vertical (right) plane before (large dots) and after (small dots) background subtraction. The statistical rms sum changes considerably due to the background $\sigma_{\text {stat }}$, while the $\sigma$ obtained from the Gaussian ( $\left.\sigma_{\text {Gauss }}\right)$ and qGaussian fit ( $\sigma_{\mathrm{qGauss}}$ ) stay unchanged. The statistical parameters and fit were calculated or performed for the average profiles. 
parameter, which does not include a parameter to model the background. As the Gaussian and q-Gaussian fit both include the parameter $c$ in Eqn. 7-8 to model the background, the obtained $\sigma$ stays almost unchanged. Therefore if absolute values are needed, it is best to subtract the background. If only relative changes are needed, it is better to not subtract the background and work with the unmodified profiles containing the full information.

\section{CALCULATION OF STATISTICAL PARAMETERS}

The statistical parameters are calculated based on the average (self .profiles_norm_avg) and moving average profiles (self.profiles_norm_mvavg) and aim at an estimate of the:

1. beam centroid

2. beam sigma

3. beam halo population

All calculated parameters param_stat are then stored in self.profiles_norm_avg_stat and self.profiles_norm_mvavg_stat, with the following format:

self.profiles_norm_mvavg_stat [plane] [slot] [param_stat]

Each parameter is calculated using different methods based on values obtained directly from the profile data or a Gaussian and q-Gaussian fit. Usually a robust estimate of e.g. the beam sigma can then be obtained by taking the average over the values obtained with all different methods. However, in particular for the beam sigma, the values differ quite significantly partly due to the background of the profiles and therefore it is better to look at the different values individually.

For the analysis the complete BSRT profiles are used and not only one side as done in [4]. It should be kept in mind that the profiles are not symmetric and all statistical parameters will therefore also be influenced by it. As this asymmetry stays rather constant along the fill (see Sec. III A, Fig. 8), it will however not influence any relative changes, which are the ones we are most interested in.

\section{A. Asymmetry of BSRT profiles}

The BSRT profiles at injection feature in general a higher amplitude on the right side of the profiles in the horizontal plane and on the left side of the profiles in the vertical plane (see Fig. 5). This can be seen even better in Fig. 8, which shows the difference between the right and left bin:

$$
R_{i}=A_{i, \text { right }}-A_{-i, \text { left }} \quad \text { with } \quad i \in\left[0, \frac{n_{\text {bins }}}{2}\right]
$$



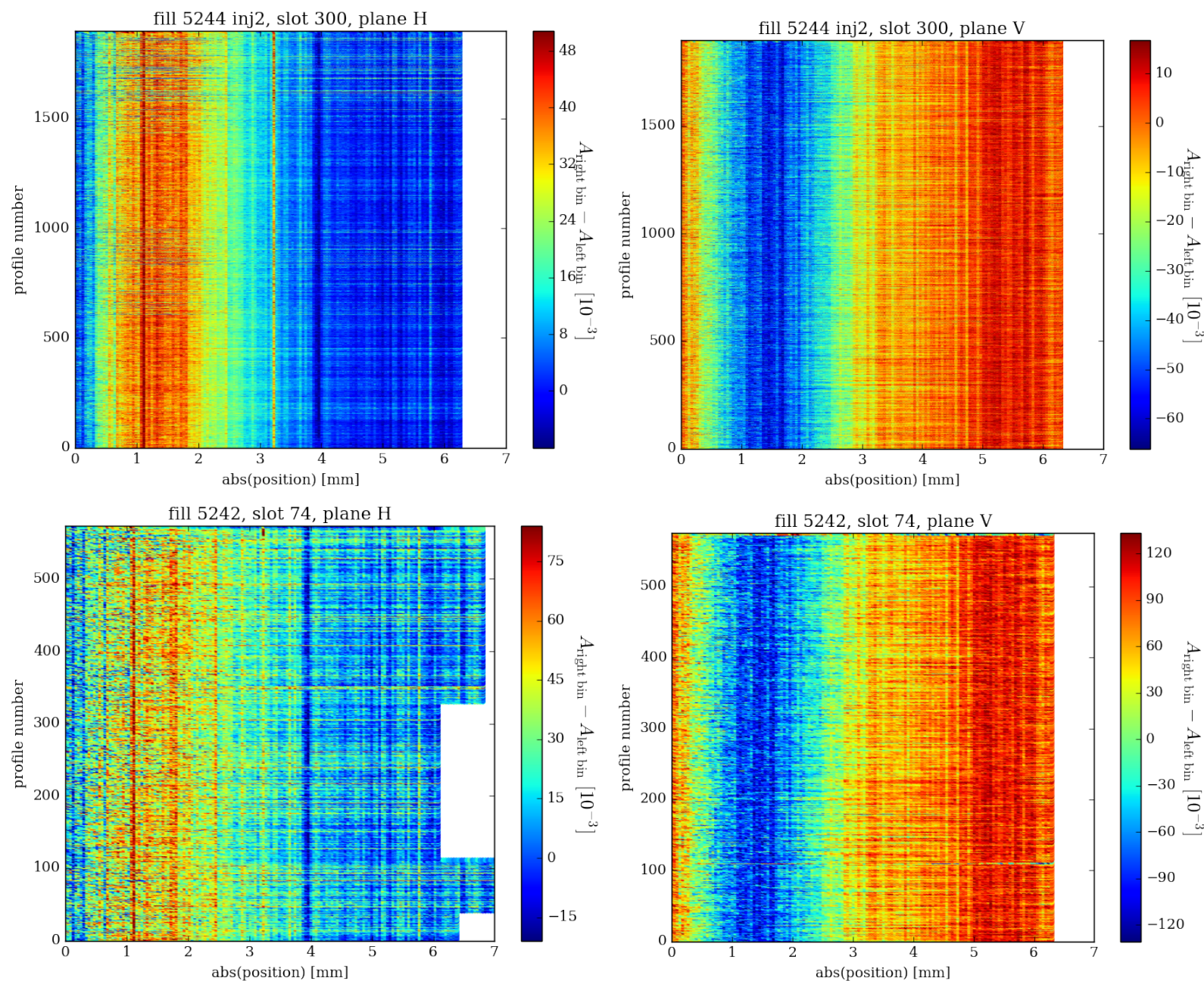

Figure 8. Difference between amplitude of left and right bin (Eqn. 11). For example the results for the BSRT profiles at injection (450 GeV) during fill 5244 for slot 300 and during fill 5242 for slot 74 are shown for the horizontal (left) and vertical (right) plane. Here the average profiles are used.

with the bins indexed between $\left[-\frac{n_{\text {bins }}}{2}, \frac{n_{\text {bins }}}{2}\right]$. This asymmetry is present in all profiles taken during MD1217 [2] and MD1415 [3]. The asymmetry can be either explained by and a constant closed orbit offset of the beam. Most likely it is however not a feature of the beam distribution but an artifact of the BSRT optics and electronics. This asymmetry is also later visible in the beam centroid (see Sec. III C, Fig. 16).

\section{B. Gaussian and q-Gaussian fit}

The average (self . profiles_norm_avg) and moving average profiles (self . profiles_norm_mvavg) are each fitted with a Gaussian distribution (light red dashed line in Fig. 1) and a q-Gaussian distribution (dark red line in Fig. 1). For both distributions, the parameter $c_{z}$ is introduced in order to model the background of the profiles implying the introduction of the parameter $a_{z}$ in order to fulfill the requirement that 
the integral over the distribution is one. The Gaussian distribution is then defined as

$$
f_{\text {Gauss }}(z):=c_{z}+a_{z} \cdot \frac{e^{-\frac{\left(z-\mu_{z}\right)^{2}}{2 \sigma_{z}^{2}}}}{\sqrt{2 \pi} \sigma_{z}}, \quad z=x, y,
$$

where $\mu_{z}$ is the mean and $\sigma_{z}$ the standard deviation of the distribution.

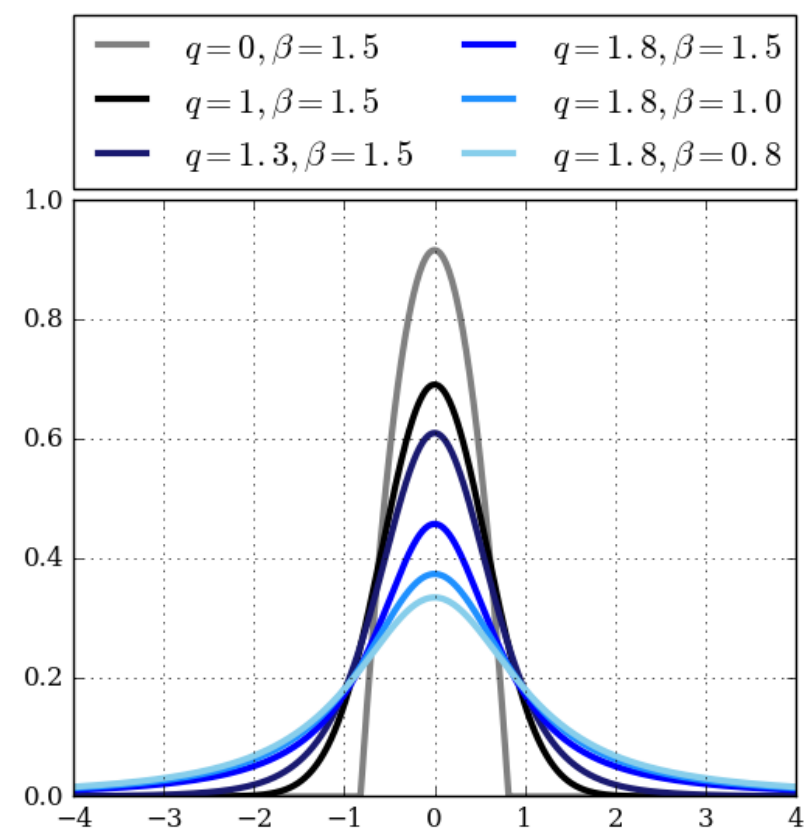

Figure 9. q-Gaussian distribution for different parameters $q$ and $\beta$.

The q-Gaussian distribution is defined as

$$
f_{\mathrm{q}-\text { Gauss }}(z)=c_{z}+a_{z} \cdot \frac{\sqrt{\beta_{z}}}{C_{q, z}} e_{q}\left(-\beta_{z}\left(z-\mu_{z}\right)^{2}\right), \quad z=x, y,
$$

where $\mu_{z}$ is the mean of the distribution and

$$
e_{q}(z)=\left(1+\left(1-q_{z}\right) \cdot z\right)^{\frac{1}{1-q_{z}}}, \quad z=x, y,
$$

is the q-exponential. The normalization factor $C_{q, z}$ is given by

$$
C_{q, z}=\left\{\begin{array}{ll}
\frac{2 \sqrt{\pi} \cdot \Gamma\left(\frac{1}{\left(1-q_{z}\right)}\right)}{\left(3-q_{z}\right) \sqrt{1-q_{z}} \cdot \Gamma\left(\frac{3-q_{z}}{2\left(1-q_{z}\right)}\right)}, & \text { for }-\infty<q<1, \\
\sqrt{\pi}, & \text { for } q=1, \\
\frac{\sqrt{\pi} \cdot \Gamma\left(\frac{3-q_{z}}{2\left(q_{z}-1\right)}\right)}{\sqrt{q_{z}-1 \cdot \Gamma\left(\frac{1}{q_{z}-1}\right)},} & \text { for } 1<q<3,
\end{array}\right\}, \quad z=x, y,
$$

and the standard deviation $\sigma_{z}$ is given by:

$$
\sigma_{z}^{2}=\left\{\begin{array}{ll}
\frac{1}{\beta\left(5-3 q_{z}\right)} & \text { for } q_{z}<\frac{5}{3} \\
\infty & \text { for } \frac{5}{3} \leq q<2 \\
\text { undefined } & \text { for } 2 \leq q<3
\end{array}\right\}, \quad z=x, y
$$


The parameter $q_{z}$ is in general an estimator for the tail population. For $q_{z} \rightarrow 1$ the Gaussian distribution is recovered, for $q_{z}>1$ the distribution features heavier tails compared to the Gaussian distribution and for $q_{z}<1$ lighter tails (see Fig. 9). For all fits, the range of the parameter $q_{z}$ is limited to $0<q_{z}<3$. Note that if the profile distribution is almost Gaussian for $q_{z} \rightarrow 1$, the q-Gaussian fit has difficulties to converge and tends to jump, otherwise it is rather stable and reliable in terms of convergence.

For both distributions, the average profiles (profs = self.profiles_norm_avg) are fitted using: scipy.optimize.curve_fit(xdata=profs ['pos'], ydata=profs ['amp'], method='lm', ...)

where $1 \mathrm{~m}$ is the Levenberg-Marquardt algorithm. In case of the moving average profiles (profs $=$ self.profiles_norm_mvavg), the standard deviation $\sigma_{A_{i}}$ of each bin is included as weights in the fit:

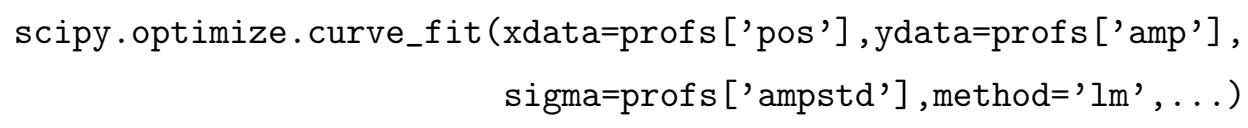

For the average profiles, the fit is not weighted as the average is only taken over a small number of profiles (here 3) leading to a large fluctuation of the standard deviation $\sigma_{A_{i}}$ of each bin. The fluctuations would then dominate the fit. All fit parameters are stored in the format c_gauss for e.g. parameter $c_{z}$ of the Gaussian distribution. The correlation $\operatorname{corr}\left(p_{i}, p_{j}\right)$ and error $\sigma_{p_{i}}$ of the fit parameters $p_{i}$ are calculated using the covariance matrix $\Sigma_{i j}=\operatorname{cov}\left(p_{i}, p_{j}\right)$ returned by curve_fit, explicitly:

$$
\begin{aligned}
\sigma_{p_{i}} & =\sqrt{\Sigma_{i i}}, \quad i=1, \ldots, n_{\mathrm{fit}} \\
\operatorname{corr}\left(p_{i}, p_{j}\right) & =\frac{\Sigma_{i j}}{\sqrt{\Sigma_{i i} \cdot \Sigma_{j j}}}, \quad i, j=1, \ldots, n_{\mathrm{fit}}
\end{aligned}
$$

The error on the fit parameters is stored in the format c_gauss_err for e.g. the error on the parameter $c_{z}$ of the Gaussian distribution. The correlation matrix is stored in pcorr_gauss and the covariance matrix in pcov_gauss for e.g. the Gaussian distribution.

The correlation of the Gaussian and q-Gaussian fit parameters is illustrated in Fig. 10-11, where fill 5242 has been used as an example. Naturally $c_{h}$ and $a_{h}$ are strongly correlated for the Gaussian as well as q-Gaussian distribution. The correlation between $\sigma_{h}$ and $c_{h}$ or respectively $a_{h}$ in case of the Gaussian distribution indicates that the background still contributes considerably to the beam sigma. The same observation holds for the q-Gaussian fit and the fit parameters $c_{h}$ and $a_{h}$ for the background and $q_{h}$ and $\beta_{h}$ for the beam sigma. The beam centroid $\mu_{h}$ is for both distributions uncorrelated. The correlation between the background and the beam sigma can be reduced but not entirely removed if the background is subtracted. As long as the correlation stays approximately constant, changes in the fit parameters are real. However, if changes in the fit parameters coincides with considerable changes in their correlation, they could be artificially introduced by a change in background or the fit itself. In this case, the background should be checked and the goodness of the fit (chi-square). 

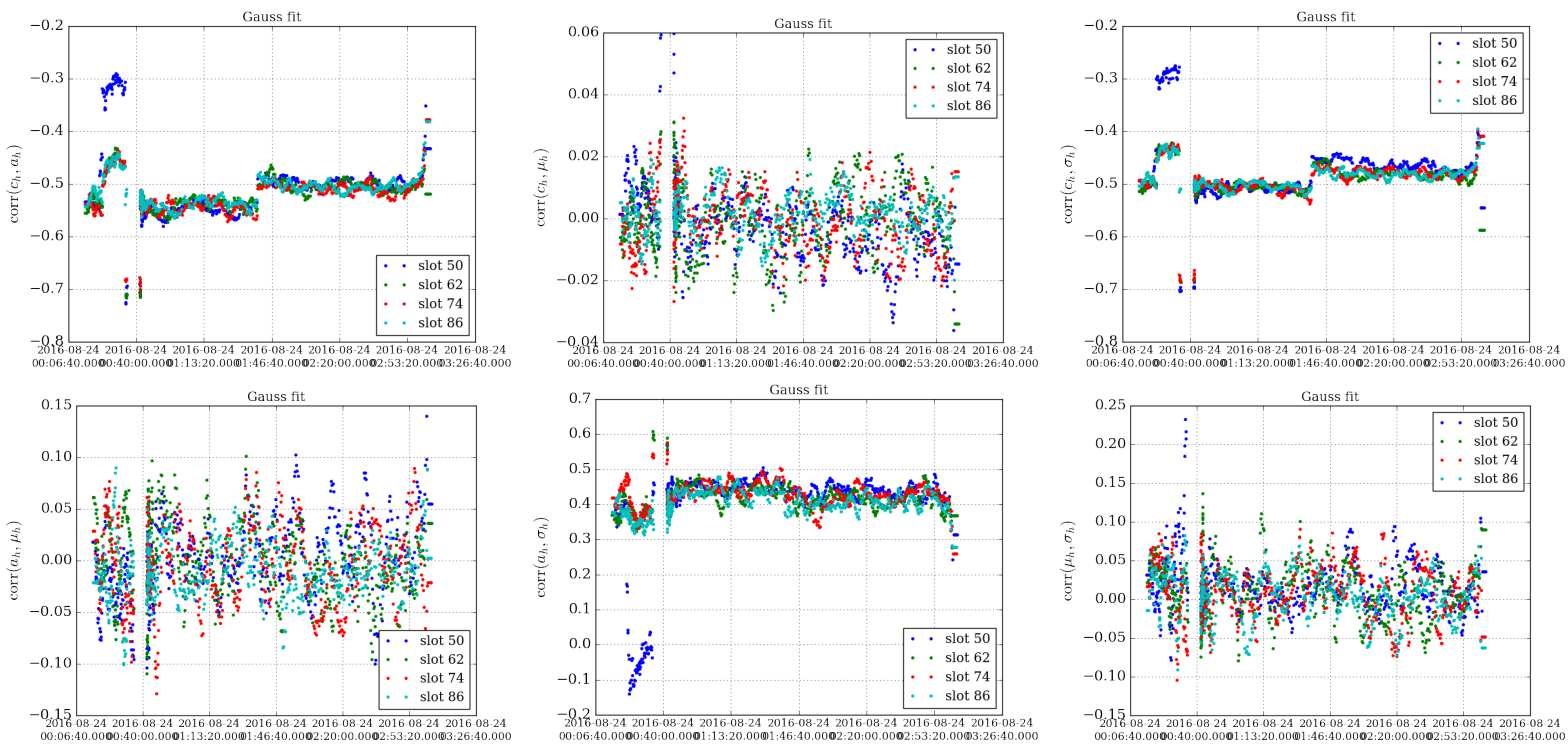

Figure 10. Correlation of Gaussian fit parameters. For example the results for the moving average profiles in the horizontal plane at injection $(450 \mathrm{GeV})$ during fill 5242 for slot 50,62,74 and 86 are shown. The background has not be subtracted.
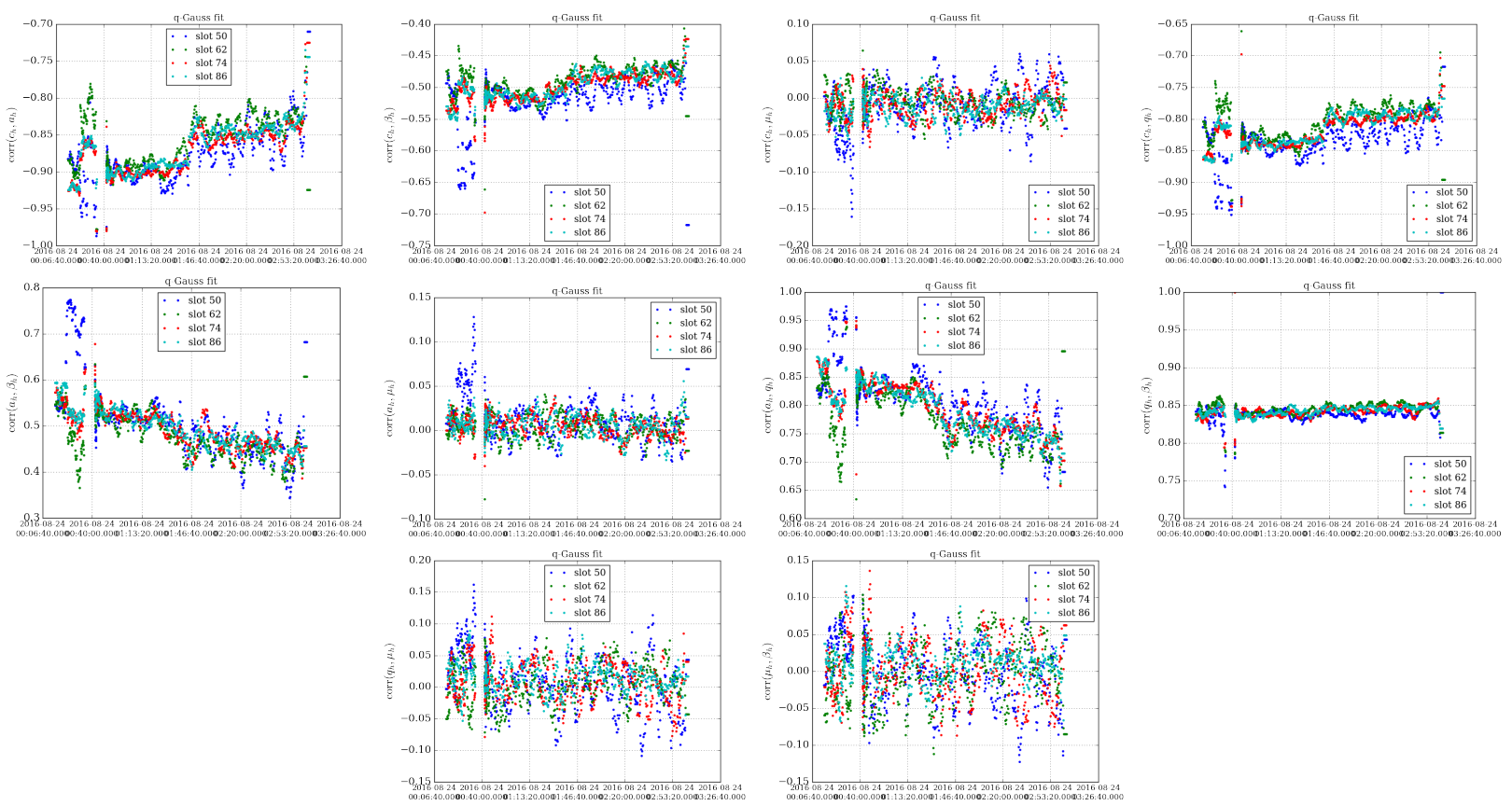

Figure 11. Correlation of q-Gaussian fit parameters. For example the results for the moving average profiles in the horizontal plane at injection ( $450 \mathrm{GeV}$ ) during fill 5242 for slot 50,62,74 and 86 are shown. The background has not be subtracted. 
The goodness of the fit is measured with the chi-square test. The normalized chi-square is given by:

$$
\chi^{2}=\frac{1}{n_{\text {bins }}-n_{\text {fit }}} \sum_{i=1}^{n_{\text {bins }}} \frac{\mu_{A_{i}}-A_{i, \text { fit }}}{\sigma_{A_{i}}}
$$

where $n_{\text {bins }}-n_{\text {fit }}$ is the degrees of freedom of the chi-square with $n_{\text {bins }}$ the number of bins and $n_{\text {fit }}$ the number of fit parameters ( 4 for Gaussian and 5 for q-Gaussian distribution), $\mu_{A_{i}}$ and $\sigma_{A_{i}}$ are the amplitude and standard deviation of bin $i$ as defined in Eqn. 3-4 and $A_{i, \text { fit }}$ is the amplitude of bin $i$ of the Gaussian or q-Gaussian fit respectively. For a good fit, the normalized $\chi^{2}$ as defined in Eqn. 20 should lie around 1. For example, the normalized $\chi^{2}$ for the q-Gaussian fit using the average and moving average profiles is shown in Fig. 12. The moving average profiles feature a $\chi^{2}$ which is smaller than expected (around 0.2-0.3) and the average profiles a $\chi^{2}$ which is by an order of magnitude too large (around 5-20). The difference from the expected value of $\chi^{2}=1$, can be due to:

1. the model does not fit the data

2. the standard deviation $\sigma_{i}$ for each bin has not been correctly estimated
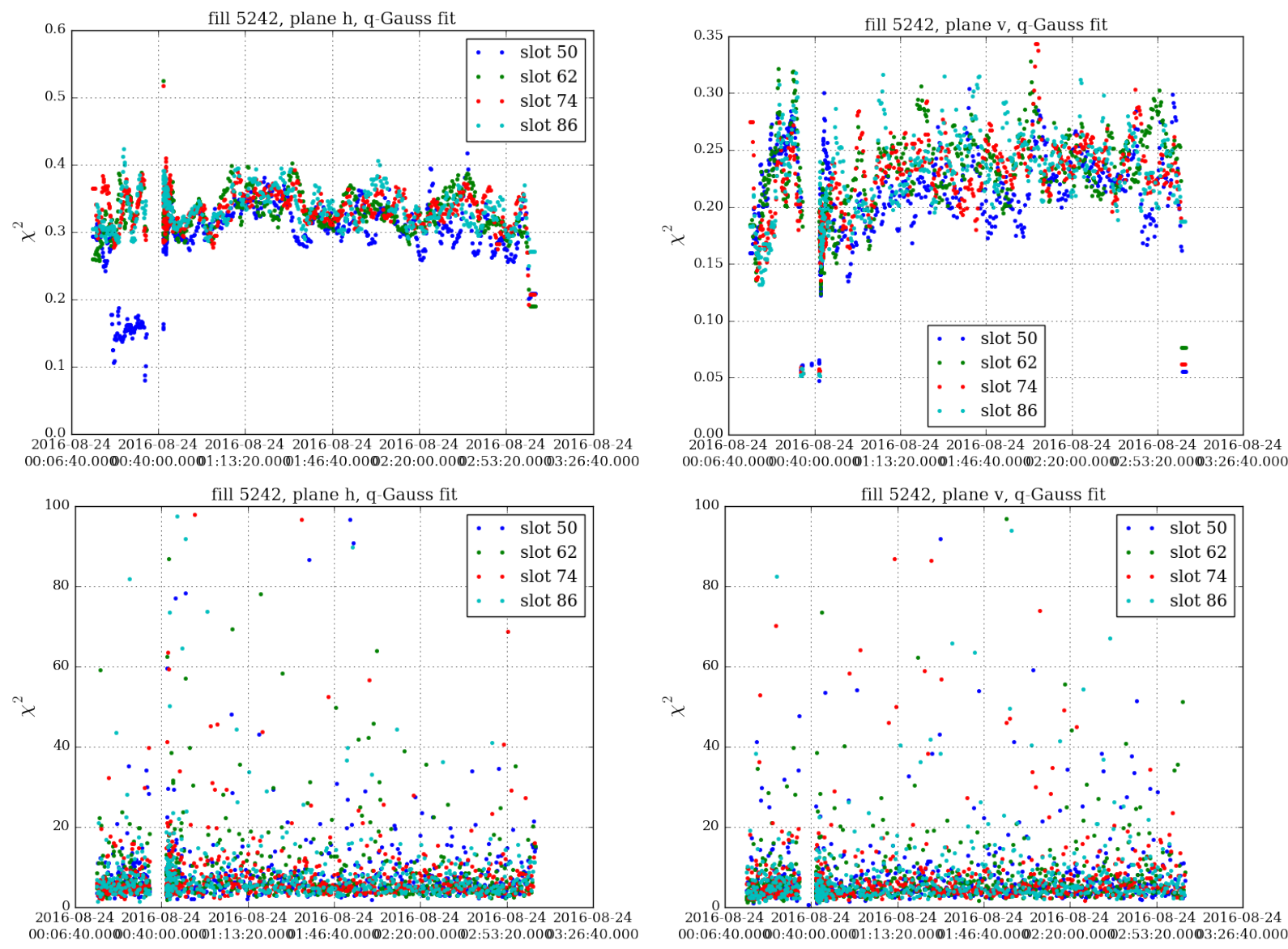

Figure 12. $\chi^{2}$ for the q-Gaussian fit using the moving average (top) and average (bottom) profiles. For example the results at injection $(450 \mathrm{GeV})$ during fill 5242 for slot 50,62,74 and 86 are shown. The background has not be subtracted. Similar values are expected also if the background is subtracted. 
In both cases, the discrepancy is most likely due to an incorrectly estimated standard deviation $\sigma_{i}$ for each bin. For the moving average profiles it is overestimated leading to a too small $\chi^{2}$, while for the average profiles it is underestimated leading to a too large $\chi^{2}$. This hypothesis is substantiated by the comparison of the $\chi^{2}$ for the Gaussian and q-Gaussian distribution using the moving average profiles (see Fig. 1213). In the vertical plane, the distribution is almost Gaussian, in which case $\chi^{2} \approx 0.2-0.25$ is obtained for both distributions. In the horizontal plane, the distribution features overpopulated tails and is thus better approximated by a q-Gaussian distribution. This is reflected in a larger $\chi^{2}$ of $\chi^{2} \approx 0.6-0.7$ for the Gaussian distribution and again $\chi^{2} \approx 0.2-0.25$ for the q-Gaussian distribution. We would therefore conclude that a $\chi^{2} \approx 0.2-0.25$ corresponds to a value of $\chi^{2} \approx 1$ due to the incorrect estimation of the standard deviation for each bin.
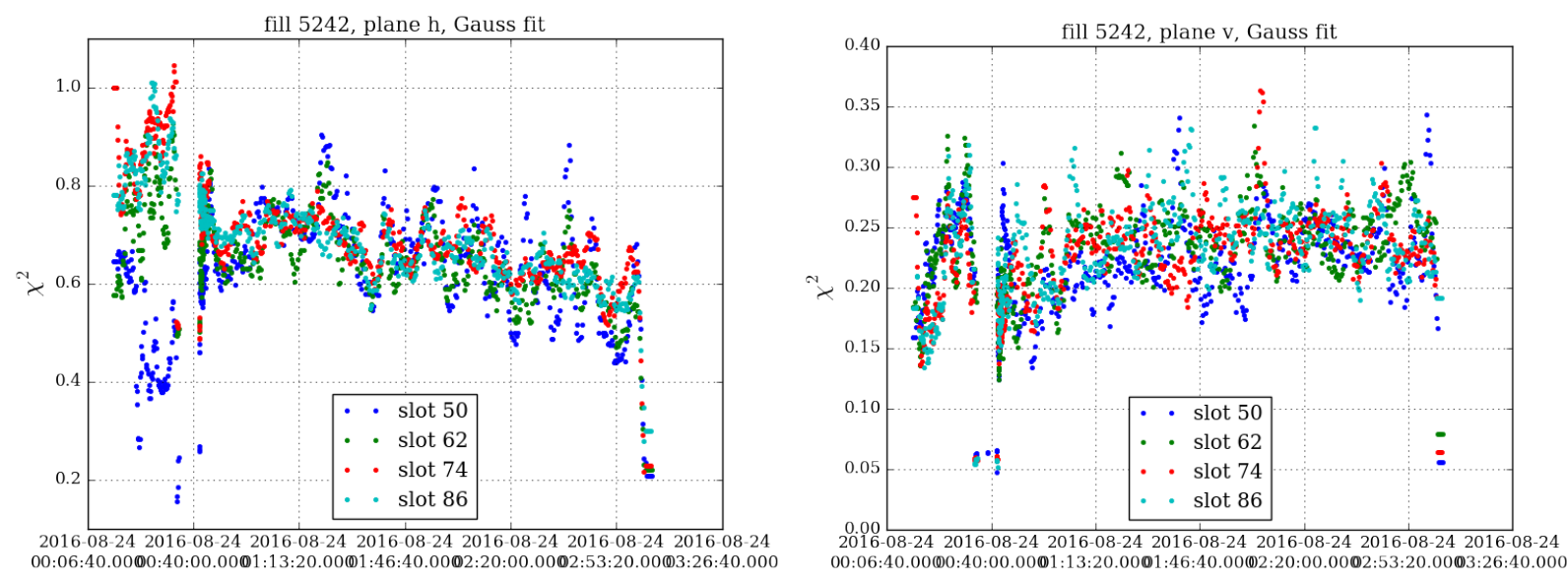

Figure 13. $\chi^{2}$ for the Gaussian fit using the moving average profiles. For example the results at injection $(450 \mathrm{GeV})$ during fill 5242 for slot 50,62,74 and 86 are shown. The background has not be subtracted. Similar values are expected also if the background is subtracted.

\section{Statistical estimate of beam centroid, beam sigma and beam halo}

In addition to the Gaussian and q-Gaussian fit, the beam centroid and beam sigma as well as the beam halo are estimated using different methods. In this section, we abbreviate the number of bins with $n_{\text {bins }}$, the bin position with $z_{i}$ and the amplitude of the normalized distribution in bin $i$ with $A_{i}$, where $A_{i}$ is either the amplitude of the average (self .profiles_norm_avg) or moving average (self .profiles_norm_mvavg) profiles.

\section{Beam centroid and beam sigma}

All estimates of the beam centroid are stored in the variables cent_*, explicitly: 
1. cent_stat is the center of gravity $\mu_{\text {stat }}$ defined by:

$$
\mu_{\mathrm{stat}}=\frac{\sum_{i=1}^{n_{\text {bins }}} z_{i} \cdot A_{i}}{\sum_{i=1}^{n_{\text {bins }}} A_{i}}
$$

2. cent_median is the median $\mu_{\text {median }}$ :

$$
\mu_{\text {median }}=\operatorname{median}(z, A)
$$

where $x$ is the array of $z_{i}$ and $A$ the array of $A_{i}{ }^{1}$

3. cent_cumsum is the position $z_{i}$ for which the cumulative sum (or integral over the distribution) takes on the value 0.5 , explicitly:

$$
\begin{aligned}
\Sigma_{i} & =\sum_{j=1}^{i}\left(z_{j}-z_{j-1}\right) \cdot A_{j}, \quad i=1, \ldots, n_{\mathrm{bins}}-1 \\
\mu_{\mathrm{cum}} & =z_{i} \text { for which } \Sigma_{i}=0.5
\end{aligned}
$$

4. cent_peak is the position $z_{i}$ for which the peak of the distribution $\mu_{\text {peak }}$ is reached.

For example the beam centroid is shown for fill 5242, slot 50 in Fig. 14.
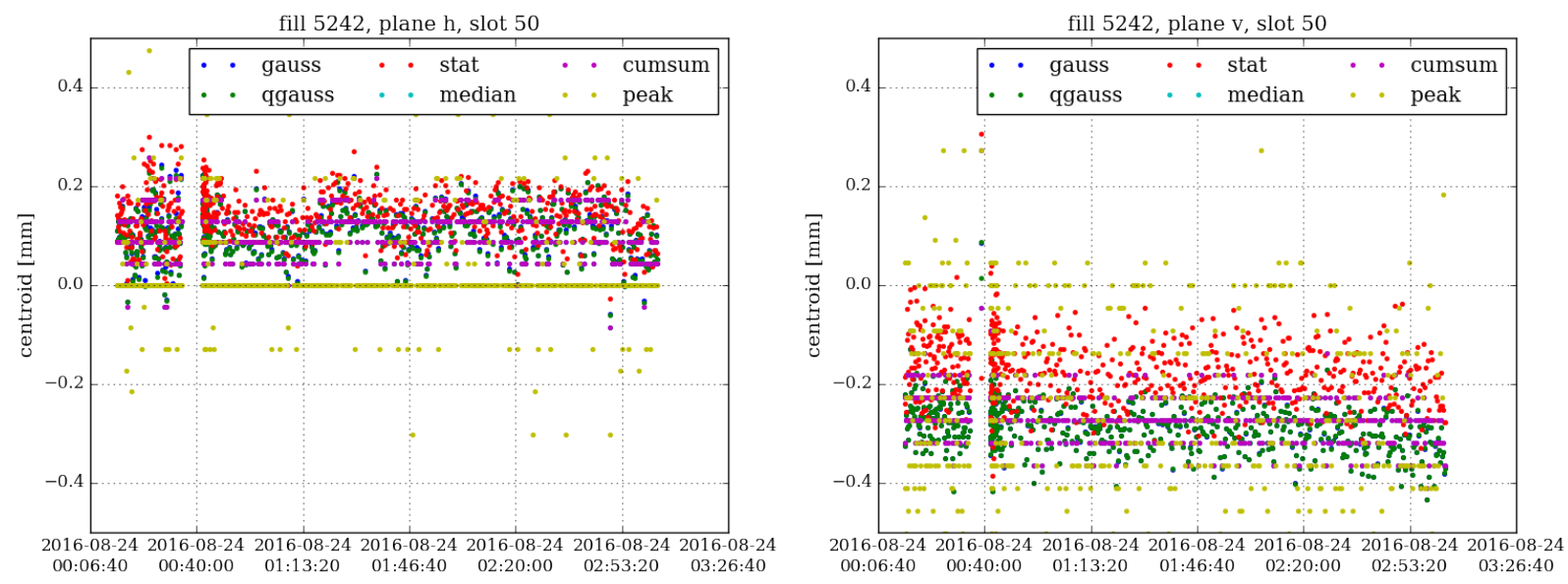

Figure 14. Bunch centroid using the average profiles. For example the results at injection $(450 \mathrm{GeV})$ during fill 5242 for slot 50 are shown. The background has not been subtracted.

All estimates of the beam sigma are stored in the variables sigma_*, explicitly:

1. sigma_stat is the statistical rms sum $\sigma_{\text {stat }}$ defined by:

$$
\sigma_{\text {stat }}=\sqrt{\frac{\sum_{i=1}^{n_{\text {bins }}} A_{i} \cdot\left(z_{i}-\mu_{\text {stat }}\right)^{2}}{\sum_{i=1}^{n_{\text {bins }}} A_{i}}}
$$

\footnotetext{
${ }^{1}$ In this implementation, the median is calculated as the 0.5 value of the cumulative sum.
} 
2. sigma_median is the sigma $\sigma_{\text {median }}$ obtained from the median absolute deviation (MAD) defined by:

$$
\sigma_{\text {median }}=1.4826 \cdot \operatorname{median}\left(\left|x-\mu_{\text {median }}\right|, A\right)
$$

where $x$ is the array of $z_{i}$ and $A$ the array of $A_{i}{ }^{1}$.

3. sigma_cumsum_* is the position $z_{i}$ for which the cumulative sum (or integral over the distribution) takes on the value $\operatorname{erf}\left(\frac{1}{\sqrt{2}}\right) \approx 0.68$ or $\left(1-\operatorname{erf}\left(\frac{1}{\sqrt{2}}\right)\right) \approx 0.32$ where erf is the error function. Explicitly:

$$
\begin{aligned}
& \sigma_{\text {cum32 }}=z_{i}-\mu_{\text {cum }} \text { for which } \Sigma_{i}=0.32 \\
& \sigma_{\text {cum68 }}=z_{i}-\mu_{\text {cum }} \text { for which } \Sigma_{i}=0.68
\end{aligned}
$$

where the definition of the beam centroid in Eqn. 21-23 has been used. For example, the beam sigma is shown for fill 5242, slot 50 in Fig. 15.
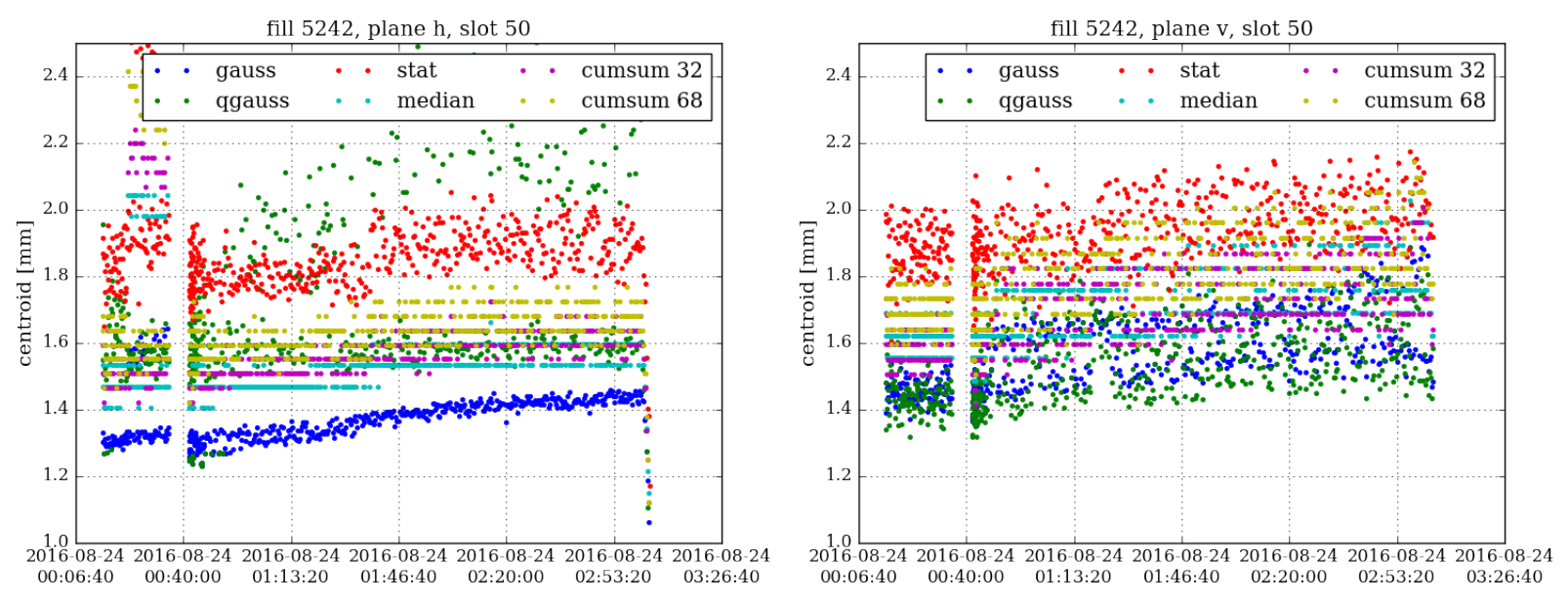

Figure 15. Bunch sigma using the average profiles. For example the results at injection $(450 \mathrm{GeV})$ during fill 5242 for slot 50 are shown. The background has not been subtracted.

To better compare the results of the beam centroid and sigma using the different methods, Fig. 16 shows a histogram of all values obtained during the entire fill 5242. As bin width the same bin width as for the profiles has been chosen. First of all, a few general observations can be made:

- The beam centroid and sigma feature very similar distributions for the 4 different bunches (slot 50, 62, 74 and 86).

- The background subtraction has a considerable impact on the calculation of the beam sigma while the beam centroid is not influenced.

For the beam centroid the following observations can be made: 

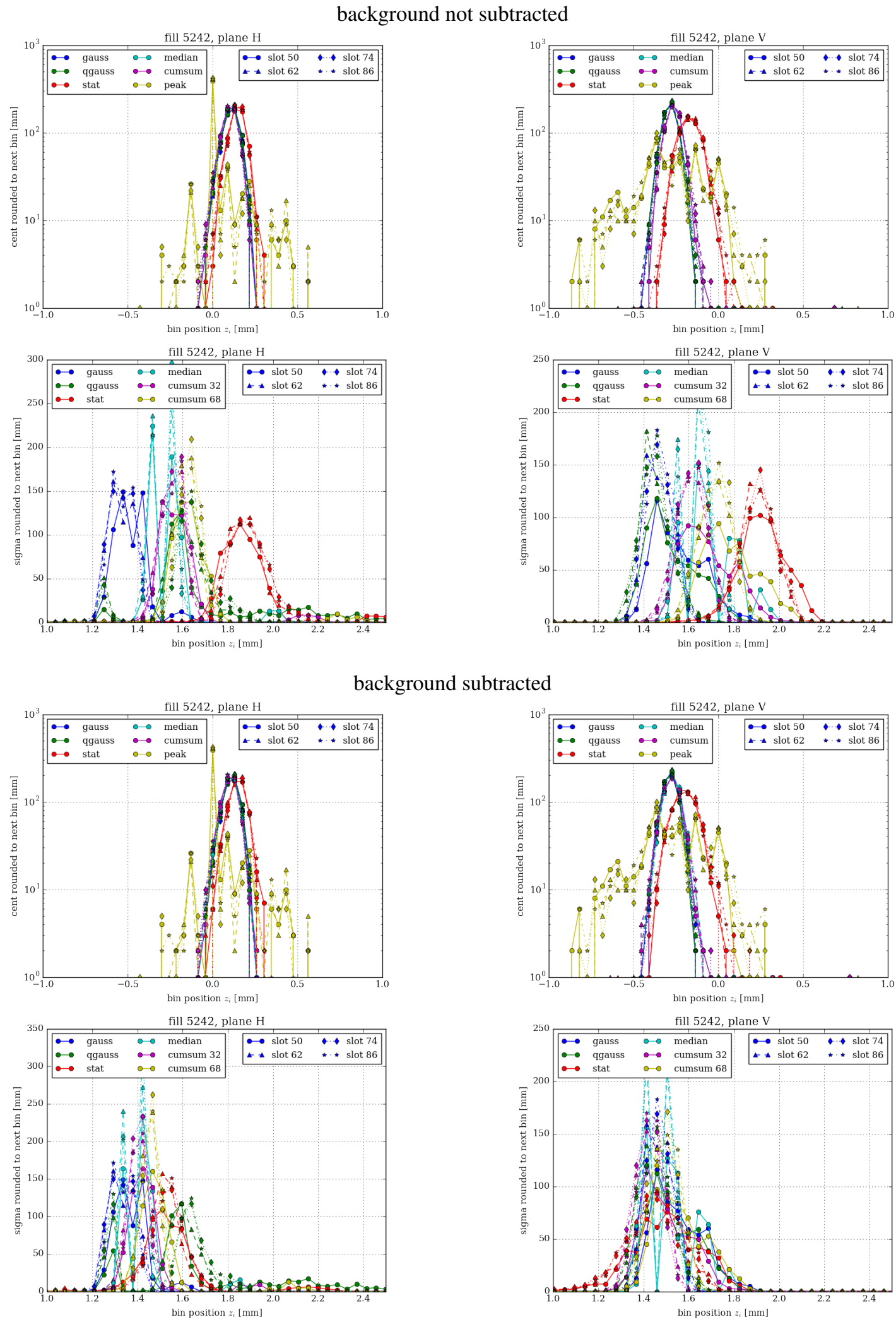

Figure 16. Histogram of the bunch centroid and sigma using the average profiles without background subtraction (top) and with background subtraction (bottom) at injection (450 GeV) during fill 5242 and for slot 50, 62, 74 and 86. Positions have been rounded to the next bin in order to generate the histogram. 
- The beam centroid as estimated with the Gaussian fit $\mu_{\mathrm{Gauss}}$, q-Gaussian fit $\mu_{\mathrm{qGauss}}$, median $\mu_{\text {median }}$ and cumulative sum $\mu_{\text {cum }}$ yield similar results for the beam centroid. The center of gravity $\mu_{\text {stat }}$ is shifted slightly to the right in comparison. The origin of this shift is unclear.

- As the profiles are in general very noisy, the peak $\mu_{\text {peak }}$ of the distribution features large jumps.

- The beam centroid is shifted to the right in the horizontal and the left in the vertical plane. This shift is either due to a real constant closed orbit offset at the location of the BSRT or it is due to a spot in the image originating from the BSRT optics and electronics (see Sec. III A).

For the beam sigma the following observations can be made:

- Without background subtraction the sigma as obtained with the different methods is heavily influenced by the background or respectively the distribution in the outermost bins of the profile. By removing the background, the sigma obtained with the different methods agrees much better.

- In the vertical plane, the distribution is almost perfectly Gaussian leading to a very good agreement of the sigma as calculated with the different methods after background subtraction.

- In the horizontal plane, the bunch distribution is non Gaussian with overpopulated tails with respect to a Gaussian distribution. This leads to a much larger spread between the different methods also after background subtraction. In particular, the sigma of the Gaussian and q-Gaussian fit differ as the q-Gaussian fit includes the $q$-parameter to better model the tails of the distribution.

- The contribution of the outermost bins (the background or noise) to the statistical rms sum $\sigma_{\text {stat }}$ is considerable. This is first of all drastically visible in a decrease of the beam sigma from around 1.9 to 1.55 just due to the background subtraction. With background subtraction, the statistical rms sum $\sigma_{\text {stat }}$ still yields larger sigma than the other methods probably due to a non-negligible contribution from the noise or background of the profiles.

\section{Beam halo}

The halo is in general difficult to estimate from the BSRT profiles as the noise level is too high. In addition to the fit parameters $c, a$ and $q$ from the Gaussian and q-Gaussian fit, information can be obtained from:

1. bg_avg* which is the mean over the left/right outermost bins and is used as estimate for the background (see Sec. II C 1):

$$
\begin{aligned}
B_{\text {left,slot }} & =\frac{1}{10} \cdot \sum_{i=10 \text { left outermost bins }} A_{i} \\
B_{\text {right,slot }} & =\frac{1}{10} \cdot \sum_{i=10 \text { right outermost bins }} A_{i}
\end{aligned}
$$


where $A_{i}$ is the amplitude of bin $i$ of the average or respectively moving average profiles.

2. sum_bin_* which is the sum over the left and right bins of high amplitude:

$$
\begin{aligned}
\Sigma_{\text {left }} & =\sum_{i \text { with }-6<z_{i}<-3} A_{i} \\
\Sigma_{\text {right }} & =\sum_{i \text { with } 3<z_{i}<6} A_{i}
\end{aligned}
$$

3. entropy which is the normalized entropy $S_{N}$, where the entropy is defined by:

$$
S=-\sum_{i=1}^{n_{\text {bins }}} A_{i} \cdot \ln \left(A_{i}\right)
$$

The normalized entropy is the entropy $S$ normalized by the maximum entropy:

$$
\begin{aligned}
S_{\max } & =-\sum_{i=1}^{n_{\text {bins }}} \frac{1}{n_{\text {bins }}} \cdot \ln \left(\frac{1}{n_{\text {bins }}}\right)=\ln \left(n_{\text {bins }}\right) \\
S_{N} & =\frac{S}{S_{\max }}
\end{aligned}
$$

For example, the parameters bg_avg*, sum_bin_*, entropy and the Gaussian and q-Gaussian fit paramter c_* are shown in Fig. 17 at injection $(450 \mathrm{GeV})$ during fill 5244. Based on this example, the background stays constant throughout the fill independent of the calculation method used. A similar value of the background is obtained from the parameter $c$ in the Gaussian and q-Gaussian fit and the average over the 10 outermost bins (bg_avg*). This is to be expected as a fit here in form of the parameter $c$ performs kind of an average of the obtained values and thus has to agree with the average over the bins bg_avg*. The other parameters are not related. In the example presented here, no considerable distribution changes are observed and thus the background stays constant throughout the fill. This might change in case of larger distribution changes [2].
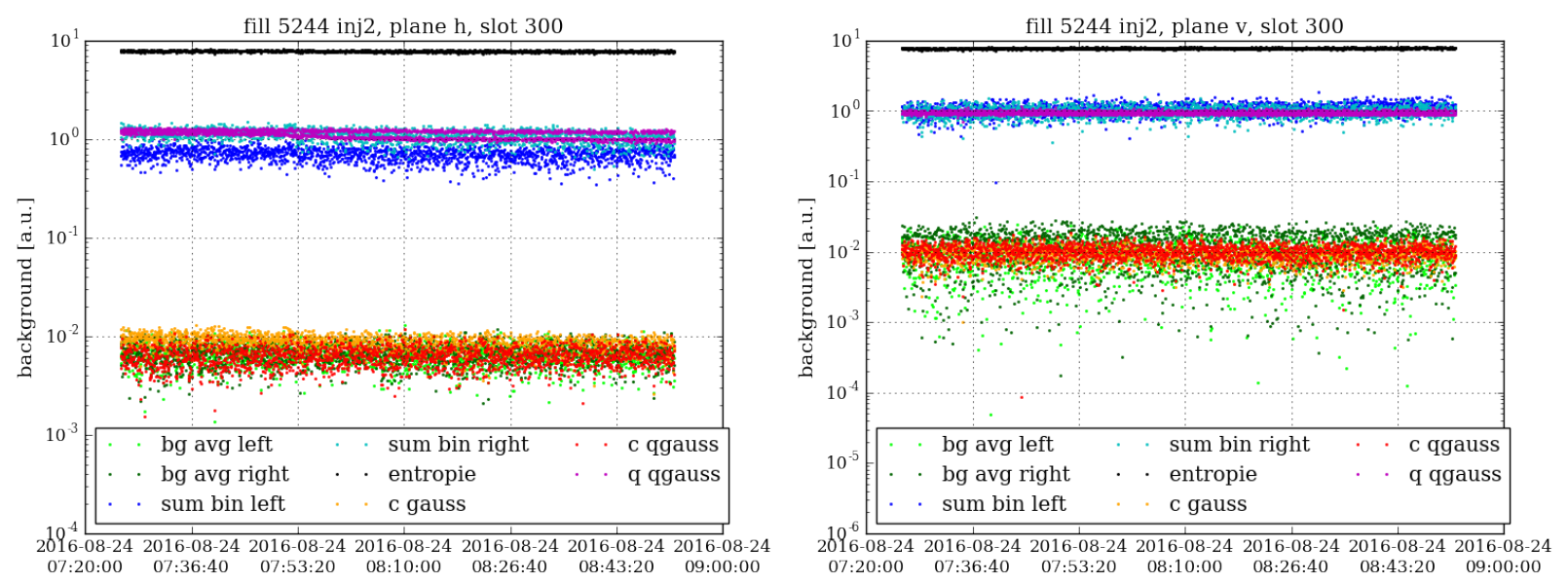

Figure 17. Estimate of beam halo at injection $(450 \mathrm{GeV})$ during fill 5244 for slot 300 using the average profiles. The background has not been subtracted. 


\section{Conversion to beam sigma and emittance}

The BSRT image formation is mathematically described as a convolution of the beam distribution with the optical resolution (LSF). Assuming that the beam distribution as well as the optical resolution (LSF) are Gaussian a conversion factor $c_{\mathrm{LSF}, z}$ ("LSF factor") can be derived which is nothing else than the width of the Gaussian distribution of the optical resolution ${ }^{2}$ :

$$
\sigma_{\text {beam }, z}=\sqrt{\sigma_{\text {profile }, z}^{2}-c_{\mathrm{LSF}, z}^{2}}, z=h, v .
$$

From the beam sigma $\sigma_{\text {beam }, z}$ the normalized emittance can then be obtained via the well known formula:

$$
\varepsilon_{N, \text { bunch }, z}=\beta_{\text {rel }} \gamma_{\text {rel }} \frac{\sigma_{\text {beam }, z}^{2}}{\beta_{\text {BSRT }, z}}, z=h, v,
$$

where $\beta_{\text {rel }}, \gamma_{\text {rel }}$ are the relativistic $\beta$ and $\gamma$, and $\beta_{\mathrm{BSRT}, z}$ is the beta function at the location of the BSRT.

In addition, the position can then also be expressed in beam sigma:

$$
z\left[\sigma_{\text {beam }, z}\right]=\frac{z[\mathrm{~mm}]}{\sigma_{\text {beam }, z}[\mathrm{~mm}]}, z=h, v .
$$

Note that the conversion from profile position to beam position is in general a non-linear. This scaling thus only represents an approximation and has to be used with care.

The beam energy, optical resolution ("LSF factor") and beta function can all be extracted from the LHC logging database, explicitly LHC.STATS:ENERGY, LHC.BSRT.5[LR]4.B[12]:LSF_[HV] and LHC.BSRT . 5 [LR] 4.B [12] :BETA_[HV].

\section{Residual and ratio of profiles}

An excellent way to analyze relative changes in the beam distribution is to plot the residual Res and ratio Rat of the profiles. Residual and ratio are automatically plotted by calling:

self.plot_all (...)

and can be combined to a video using:

self.mk_profile_video(...)

The residual Res and the ratio Rat for bunch $i$ at time stamp $t_{i}$ with respect to reference bunch $j$ at time stamp $t_{j}$ is defined by

$$
\begin{aligned}
\operatorname{Res}\left(t_{i}, z\right) & =\mu_{A_{i}}\left(t_{i}, z\right)-\mu_{A_{j}}\left(t_{j}, z\right), \quad z=h, v \\
\operatorname{Rat}\left(t_{i}, z\right) & =\frac{\mu_{A_{i}}\left(t_{i}, z\right)}{\mu_{A_{j}}\left(t_{j}, z\right)}, \quad z=h, v
\end{aligned}
$$

\footnotetext{
2 The convolution of two uni-variate Gaussian distributions $\mathrm{f}$ and $\mathrm{g}$ having respectively the means $\mu_{f}$ and $\mu_{g}$ and standard deviation $\sigma_{f}$ and $\sigma_{g}$ is a Gaussian distribution with mean and standard deviation $\mu_{f * g}=\mu_{f}+\mu_{g}$ and $\sigma_{f * g}=\sqrt{\sigma_{f}^{2}+\sigma_{g}^{2}}$
} 
where $\mu_{A_{i}}\left(t_{i}, z\right)$ denotes the average or moving average amplitude at bin $z$ of bunch $i$ at time stamp $t_{i}$. The error on the residual $\sigma_{\mathrm{Res}}\left(t_{i}, z\right)$ and ratio $\sigma_{\mathrm{Rat}}\left(t_{i}, z\right)$ of bin $i$ are calculated by error propagation of the error on the mean amplitude $\sigma_{\mu_{A_{i}}}\left(t_{i}, z\right)$ of bunch $i$ at time stamp $t_{i}$ as defined in Eqn. 5 (e.g. self.profiles_norm_avg['amperr']):

$$
\begin{aligned}
& \sigma_{\text {Res }}\left(t_{i}, z\right)=\sqrt{\sigma_{\mu_{A_{i}}}\left(t_{i}, z\right)^{2}+\sigma_{\mu_{A_{j}}}\left(t_{j}, z\right)^{2}}, \quad z=h, v \\
& \sigma_{\text {Rat }}\left(t_{i}, z\right)=\operatorname{abs}\left(\frac{\mu_{A_{i}}\left(t_{i}, z\right)}{\mu_{A_{j}}\left(t_{j}, z\right)}\right) \cdot \sqrt{\left(\frac{\sigma_{\mu_{A_{i}}}\left(t_{i}, z\right)}{\mu_{A_{i}}\left(t_{i}, z\right)}\right)^{2}+\left(\frac{\sigma_{\mu_{A_{j}}}\left(t_{j}, z\right)}{\mu_{A_{j}}\left(t_{j}, z\right)}\right)^{2}, \quad z=h, v}
\end{aligned}
$$

The residual and ratio can be either taken with respect to the initial distribution of the bunch itself or with respect to another bunch.

The residual is in general more sensitive to changes in the core of the distribution while the ratio is more sensitive to changes in the high amplitude tails. In simulations, both parameters proved to be very useful [6]. In case of the BSRT, the profiles are too noisy to yield useful results for the ratio, but very good results can be obtained using the less noise sensitive residual. In particular, if the average over many profiles is taken, the noise can be considerably reduced and relative changes in the beam distribution can be detected and visualized. As an example, Fig. 18 shows the change of a bunch (slot 1300) after a resonant excitation (10th turn V pulsing) during fill 5243 (see [2]). In this case, a depletion of the core and an increase of the tails is visible (black line in Fig. 18) in the residual and also the ratio. The error on the profiles, the residual and the ratio is indicated with an gray envelope.
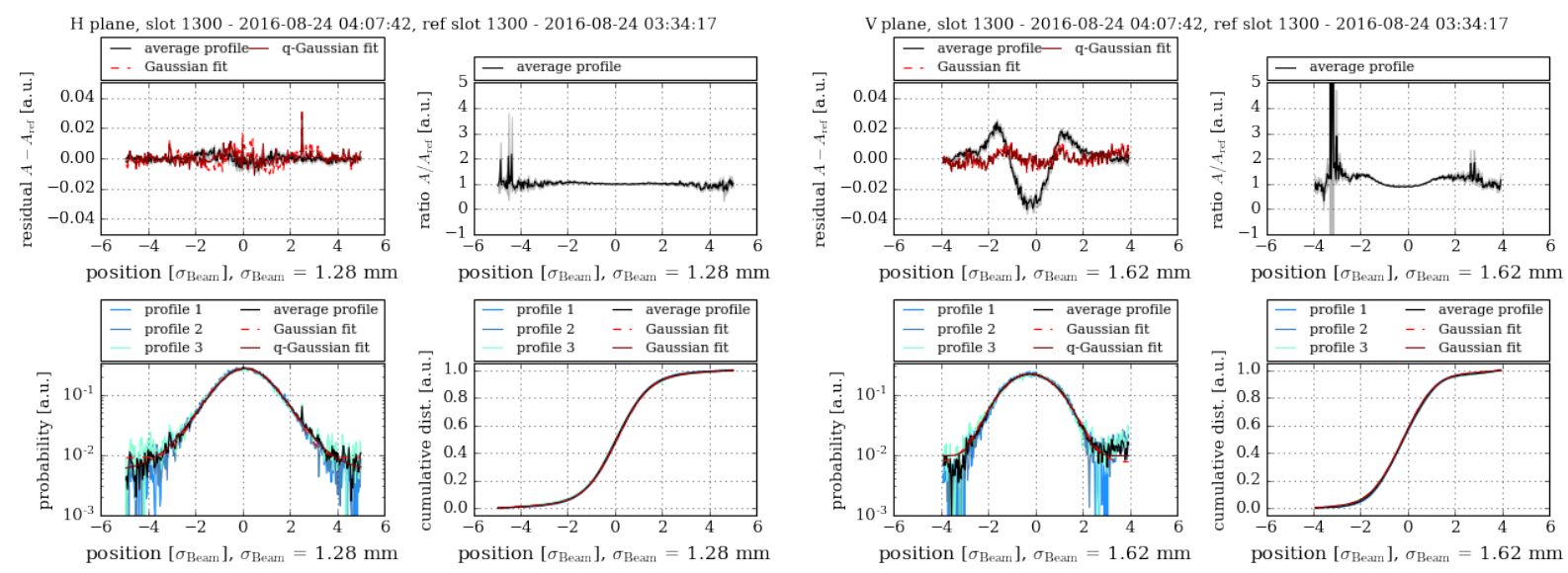

Figure 18. BSRT profile of an excited bunch (10th turn $\mathrm{V}$ pulsing) for the horizontal (left) and vertical (right). The residual and ratio are taken with respect to a profile of the bunch itself well before the excitation is switched on. The background is not subtracted and the moving average profiles over 11 time stamps (33 profiles) are shown. The position is expressed in beam sigma using the conversion of Eqn. 38.

In addition to the residual and ratio with respect to another profile, also the residual with respect to the Gaussian $\left(\operatorname{Res}_{\text {Gauss }}\left(t_{i}, z\right)\right)$ and q-Gaussian fit $\left(\operatorname{Res}_{\mathrm{qGauss}}\left(t_{i}, z\right)\right)$ of the bunch itself during the same time stamp 
is calculated and shown in red and dark red in Fig. 18. The residual with respect to the Gaussian fit is explicitly defined by:

$$
\operatorname{Res}_{\text {Gauss }}\left(t_{i}, z\right)=\mu_{A_{i}}\left(t_{i}, z\right)-f_{\text {Gauss }, i}\left(t_{i}, z\right), \quad z=h, v
$$

where $\mu_{A_{i}}\left(t_{i}, z\right)$ is the average amplitude of bunch $i$ at time stamp $t_{i}$ and $f_{\text {Gauss }, i}\left(t_{i}, z\right)$ is the amplitude as obtained with the Gaussian fit for bunch $i$ at time stamp $t_{i}$. The residual with respect to the q-Gaussian distribution is defined in the same way. Taking the example shown in Fig. 18, a significant deviation from a Gaussian distribution is seen in the horizontal plane and from both fits in the vertical plane.

\section{SUMMARY}

This note describes the analysis of the BSRT profiles as implemented in [1] based on the profiles taken at injection with $E_{\text {beam }}=450 \mathrm{GeV}$ in MD1217 [2] and MD1415 [3]. The profile analysis consists in summary of the following steps:

1. Discarding profiles containing only noisy profiles (see Sec. II A).

2. Normalizing profiles so that the integral over the distribution is one. By this normalization, all profiles have approximately the same peak amplitude. To reduce the noise, the profiles of each time stamp are averaged and in addition a moving average over several time stamps is taken (see Sec. II B).

3. The averaged and moving averaged profiles are fitted with a Gaussian and q-Gaussian distribution (see Sec. III B). In addition, the beam centroid and beam sigma are calculated using different methods (see Sec. III C).

4. The residual and ratio of the distribution with respect to an user defined other bunch and time stamp are calculated. Usually the residual and ratio is calculated with respect to the initial bunch distribution or to another reference bunch (see Sec. III D).

In particular the q-Gaussian fit and the evolution of the residual and ratio of the distribution proved to be particularly useful in analyzing distribution changes $[2,3]$.

\section{ACKNOWLEDGMENTS}

We want to thank in particular Georges Trad and Enrico Bravin for their support and explanations of the BSRT. We also want to thank all MD participants of MD1217 and MD1415 who helped take the data shown in this note. 
[1] Miriam Fitterer, "Python class for BSRT profile analysis," (2016).

[2] Miriam Fitterer, Giulio Stancari, Alexander Valishev, Roderik Bruce, Parthena Stefania Papadopoulou, Giulia Papotti, Dario Pellegrini, Stefano Redaelli, Georges Trad, Daniel Valuch, Gianluca Valentino, Joschka Wagner, and Chen Xu, Effect of a resonant excitation on the evolution of the beam emittance and halo population, Tech. Rep. CERN-ACC-NOTE-2017-0037 (2017).

[3] Miriam Fitterer, Roderik Bruce, , Wolfgang Höfle, Michael Hostettler, Parthena Stefania Papadopoulou, Giulia Papotti, Yannis Papaphilippou, Dario Pellegrini, Giulio Stancari, Georges Trad, Daniel Valuch, Gianluca Valentino, Alexander Valishev, Joschka Wagner, and Chen Xu, MD1271: Effect of low frequency noise on the evolution of the emittance and halo population, Tech. Rep. to be published (CERN, 2017).

[4] Stefania Papadopoulou, Fanouria Antoniou, Theodoros Argyropoulos, Miriam Fitterer, Michael Hostettler, and Yannis Papaphilippou, "Modelling and measurements of bunch profiles at the LHC," Conf. Proc. IPAC'17 (2017).

[5] Rhodri Jones, "Status of beam instrumentation," (2016), 6th HL-LHC Collaboration Meeting.

[6] Miriam Fitterer, Giulio Stancari, and Alexander Valishev, Effect of pulsed hollow electron-lens operation on the proton beam core in LHC, Tech. Rep. FERMILAB-TM-2635-AD (Fermilab, 2016). 